\title{
Downregulation of hsa_circ_0074854 Suppresses the Migration and Invasion in Hepatocellular Carcinoma via Interacting with HuR and via Suppressing Exosomes-Mediated Macrophage M2 Polarization
}

\author{
Yong Wang ${ }^{l *}$ \\ Rongfen $\mathrm{Gao}^{2}$ ** \\ Jinpeng $\mathrm{Li}^{3, *}$ \\ Shaotao Tang' \\ Shuai $\mathrm{Li}^{\mathrm{I}}$ \\ Qiangsong Tong' \\ Shiwang $\mathrm{Li}^{1}$
}

'Department of Pediatric Surgery, Union Hospital, Tongji Medical College,

Huazhong University of Science and Technology, Wuhan, 430022, Hubei, People's Republic of China; ${ }^{2}$ Department of Rheumatology and Immunology, Tongji Hospital, Tongji Medical College, Huazhong University of Science and Technology, Wuhan, 430030, Hubei, People's Republic of China; ${ }^{3}$ Department of Thyroid and Breast Surgery, Wuhan University Zhongnan Hospital, Wuhan, 43007I, Hubei, People's Republic of China

*These authors contributed equally to this work
This article was published in the following Dove Press journal: International Journal of Nanomedicine

Background: Circular RNAs (circRNAs) have been identified as key factors in the development of hepatocellular carcinoma (HCC). However, the role and potential molecular mechanism of circRNAs in HCC remain largely unclear. In addition, exosomes are known as important messengers of the cross-talk between tumor cells and immune cells, while the role of extracellular circRNAs in the cell-to-cell communication of tumor cells and immune cells remains not unclear.

Methods: The level of hsa_circ_0074854 in HCC cell lines and HCC cell-derived exosomes was assessed using RT-qPCR assay. In addition, CCK-8 and transwell assays were used to determine the viability, migration and invasion of $\mathrm{HCC}$ cells.

Results: Hsa_circ 0074854 expression was upregulated in HCC tissues and HCC cell lines. Additionally, hsa circ 0074854 knockdown was found to inhibit HCC growth in vitro and in vivo. Mechanistically, hsa_circ_0074854 knockdown inhibited the migration and invasion of $\mathrm{HCC}$ cells via interacting with human antigen $\mathrm{R}(\mathrm{HuR})$ to reduce its stability. Furthermore, hsa_circ_0074854 can be transferred from HCC cells to macrophages via exosomes. Exosomes with downregulated hsa_circ_0074854 suppressed macrophage M2 polarization, which in turn suppressing migration and invasion of HCC cells both in vitro and in vivo.

Conclusion: Downregulation of hsa_circ_0074854 suppresses the migration and invasion in hepatocellular carcinoma via interacting with $\mathrm{HuR}$ and via suppressing exosomes-mediated macrophage M2 polarization. Collectively, these findings may help to understand the diagnosis and treatment of HCC.

Keywords: circular RNAs, hepatocellular carcinoma, exosomes, macrophage polarization, invasion

\section{Introduction}

Hepatocellular carcinoma (HCC) is responsible for the third leading cause of cancer deaths worldwide, causing over 600, 000 deaths annually. ${ }^{1,2}$ Hepatitis B virus (HBV) and hepatitis $\mathrm{C}$ virus (HCV) infection, non-alcoholic fatty liver disease, diabetes and alcohol abuse are the important risk factors in the progression of HCC. $^{3-5}$ Many therapy strategies have been applied to treat HCC, including drug intervention, conservative, surgical and chemoembolization treatment. ${ }^{6}$
Correspondence: Yong Wang

Department of Pediatric Surgery, Union Hospital, Tongji Medical College,

Huazhong University of Science and

Technology, No. 1277 Jiefang Avenue,

Wuhan, Hubei, 430022, People's Republic of China

Email wangyong_678@I26.com 
Unfortunately, most HCC patients are often diagnosed in an advanced stage with a worse long-term prognosis, due to delayed diagnosis and treatment. ${ }^{7}$ However, the fiveyear survival rate of patients with $\mathrm{HCC}$ is $>70 \%$ if patients are diagnosed at the earliest stage. ${ }^{8}$ Thus, exploring the promising tumor-related markers may be useful for early diagnosis of HCC.

Circular RNAs (circRNAs) are a group of non-coding RNAs that are characterized by a covalently closed loop structure lacking a cap structure at $5^{\prime}$ end and a poly(A) tail at the $3^{\prime}$-end. ${ }^{9,10}$ CircRNAs were identified to be critical regulators in HCC. ${ }^{11}$ For instance, circRNA_0000502 inhibited apoptosis and promoted metastasis of HCC cells via targeting microRNA-124. ${ }^{12}$ Huang et al found that exosomal circRNA-100338 promoted cell metastasis in HCC. ${ }^{13}$ However, the role and molecular mechanism of circRNAs in HCC need to be further investigated.

Exosomes are small (40-150 $\mathrm{nm})$ extracellular vesicles. ${ }^{14}$ Exosomes carry a group of specific proteins, lipids, DNA, RNA, miRNA, lncRNA and circRNA and are then transported to adjacent cells or distant cells. ${ }^{15,16}$ Evidences have been shown that exosomes play a key role in cell-to-cell communication in the tumor microenvironment (TME). ${ }^{17,18}$ Tumor cell-derived exosomes have been reported to play an important role in promoting tumor invasion and metastasis. ${ }^{19-21}$ For example, circular RNA IARS can be transported by exosomes from pancreatic cancer cells to endothelial cells, thereby promoting tumor invasion and metastasis. ${ }^{22}$ However, the relationship between exosomal circRNAs and invasion in HCC remains largely unclear.

Tumor-associated macrophages (TAMs) are the most abundant immune cells in TME to facilitate tumor migration and invasion. ${ }^{23,24}$ In general, macrophages can be polarized into M1 (classically activated) or M2 (alternatively activated) macrophages depending on environmental cues. ${ }^{25}$ M1-polarized macrophages are activated by cytokines including IFN $\gamma$ and LPS, and M2-polarized macrophages are activated by IL-4 and IL-13. ${ }^{26} \mathrm{M} 1-$ polarized macrophages play an anti-tumor role, whereas M2-polarized macrophages are thought to promote tumor growth. $^{27}$ It has been shown that TAMs closely resemble the M2-polarized macrophages. ${ }^{26}$ Meanwhile, TAMs with an M2 phenotype can promote cancer progression and metastasis. $^{28,29}$ However, the communication between tumor cells and TAMs remains largely unknown in HCC.

In this study, we found that hsa_circ_0074854 is upregulated in HCC cells, and downregulation of hsa_circ_0074854 inhibited the migration and invasion of HCC cells. Mechanistically, hsa_circ_0074854 knockdown could reduce the stability of human antigen $\mathrm{R}$ (HuR) protein and then suppress epithelial-mesenchymal transition (EMT) to inhibit migration and invasion. In addition, oligonucleotide sequences (hsa_circ_0074854shRNA1) can be transferred by exosomes to macrophages to suppress macrophage M2 polarization. Meanwhile, inhibition of M2-type macrophage of TAMs prevented the migration and invasion of HCC cells in vitro and in vivo. These findings may help to understand the diagnosis and treatment of HCC.

\section{Materials and Methods Microarray Data Analysis}

HCC-related microarray datasets (GSE94508, GSE97332) were downloaded from the Gene Expression Omnibus database (https://www.ncbi.nlm.nih.gov/geo/). R language was performed to screen out the differentially expressed circRNAs (DEcircRNAs) between HCC tissues and normal tissues. The circRNAs with $\mathrm{P}<0.01$ and $|\log 2(\mathrm{FC})|>2$ were selected as the significantly DEcircRNAs. The intersection of DEcircRNAs from two datasets was performed using the Venn Diagram package.

\section{Cell Culture}

HCC cell lines (HepG2, Huh-7, Hep3B2.1-7, HCCLM3), normal human hepatic cell line L02 and monocytic cell line THP-1 were obtained from the Type Culture Collection of the Chinese Academy of Sciences (Shanghai, China). Cell lines were maintained in DMEM (Thermo Fisher Scientific) containing $10 \%$ FBS and kept under $5 \% \mathrm{CO}_{2}$ at $37^{\circ} \mathrm{C}$. To induce monocyte differentiation into macrophages, THP-1 cells were incubated with phorbol-12 myristate-13 acetate (PMA; $100 \mathrm{ng} / \mathrm{mL}$ ) for $24 \mathrm{~h}$.

\section{Reverse Transcription Quantitative Real-Time PCR}

RNA was extracted from cells using TRIzol reagent (Thermo Fisher Scientific). Complementary DNA (cDNA) was synthesized using an EntiLink ${ }^{\mathrm{TM}}$ 1st Strand cDNA Synthesis Kit (Wuhan, China). After that, the qPCR was performed on a StepOne ${ }^{\mathrm{TM}}$ Real-Time PCR System (Thermo Fisher Scientific) with the EnTurbo ${ }^{\text {TM }}$ SYBR Green PCR SuperMix (Wuhan, China). Primer sequences were listed as in Table 1. $\beta$-actin works as an internal control. 


\section{Lentivirus Infection and Construction of Stable Cell Lines}

The oligonucleotides to suppress hsa_circ_0074854 or HuR expression and the oligonucleotides to overexpress HuR expression were designed and obtained by Ribobio (Guangdong, China) respectively. These oligonucleotides were cloned into lentiviral expression vector pLVX-IRES-Puro (Clontech Laboratories). After that, $293 \mathrm{~T}$ cells were transfected with vectors described above, and lentivector packaging plasmid mix (pLP/ VSVG, pLP1, and pLP2) using Lipo2000 (Thermo Fisher Scientific). Later on, the supernatant containing infectious lentivirus was collected at $48 \mathrm{~h}$, and then filtered through a $0.22 \mu \mathrm{m}$ filter membrane to obtain viral particles. Next, the viral supernatant was added into the plate to infect HepG2 or Huh-7 cells. Seventytwo hours after transduction, the infected cells were selected by puromycin $(2 \mu \mathrm{g} / \mathrm{mL}$, Amresco, Cleveland, $\mathrm{OH}$, USA).

The oligonucleotide sequences were listed as followed: hsa_circ_0074854-shRNA1: 5'- GGATGCTTCTGGGAA TTTATTCAAGAGATAAATTCCCAGAAGCATCCTTTTT-3'; hsa_circ_0074854-shRNA2: 5'-GGCATGTGCAAT TGCAGATTTCAAGAGAATCTGCAATTGCACATGCCTTTTT-3'; HuR-shRNA1: 5'-TCGGGAGAACGAATTT GATCGTCAATTCAAGAGATTGACGATCAAATTCGTTCTCCCGATTTTT-3'; HuR-shRNA2: 5'- CGGGATAA AGTAGCAGGACACAGCTTTCAAGAGAAGCTGTGTCCTGCTACTTTATCCCGTTTTT-3'.

\section{Cell Viability Assay}

$5 \times 10^{3}$ HepG2 or Huh-7 cells were plated onto 96-well plates and were incubated for $24 \mathrm{~h}, 48 \mathrm{~h}$ and $72 \mathrm{~h}$. After that, $10 \mu \mathrm{L}$ of Cell Counting Kit-8 (CCK-8; Dojindo) reagent was added into each well and incubated for another $2 \mathrm{~h}$. Subsequently, the absorbance values at 450 $\mathrm{nm}$ were measured using a SpectraMax microtiter plate reader (Molecular Devices).

\section{Cell Apoptosis Assay}

Annexin V-FITC Apoptosis Staining/Detection kit (Thermo Fisher Scientific) was utilized to assess cell apoptosis according to the manufacturer's instructions. Apoptotic cells were analyzed by a flow cytometer (BD Biosciences) using BD FACSDiva software (BD Biosciences).

\section{Western Blot Assay}

Enhanced BCA Protein Assay kit (Beyotime) was used to quantify the protein concentration. The protein was separated by SDS-PAGE and then transferred onto polyvinylidene difluoride (PVDF) membranes. After that, the membranes were probed with antibodies against Bcl-2 (1:1000, Abcam Cambridge, MA, USA), active caspase 3 (1:1000, Abcam), PDCD5 (1:1000, Abcam), E-cadherin (1:1000, Abcam), $\alpha$ SMA (1:1000, Abcam), Vimentin (1:1000, Abcam), HuR (1:1000, Abcam), ZEB-1 (1:1000, Abcam), $\beta$-actin (1:1000, Abcam) at $4^{\circ} \mathrm{C}$ overnight, following by incubation with the corresponding secondary antibodies (1:5000, Abcam) at room temperature for $1 \mathrm{~h}$. Subsequently, the blots were developed by enhanced chemiluminescence (Thermo Fisher Scientific). ImageJ software was used to quantify the protein bands.

\section{RNA Pull-Down Assay}

Biotinylated hsa_circ_0074854 was obtained from GenePharma (Shanghai, China). HepG2 cells were transfected with biotinylated hsa_circ_0074854 for $24 \mathrm{~h}$. After that, cell lysates were cultured with streptavidin-coated magnetic beads (Thermo Fisher Scientific). The biotincoupled RNA complex was pulled down and the level of HuR was detected by RT-qPCR.

\section{Fluorescence in situ Hybridization (FISH)} The FISH assay was performed in HepG2 cells as described previously. ${ }^{30}$ The fluorescence-conjugated hsa_circ_0074854 or HuR mRNA probes was designed and obtained by RiboBio (Guangzhou, China). Nucleus was counterstained with DAPI. The image was captured by a Leica confocal microscope (Leica Microsystems, Mannheim, Germany).

\section{Exosome Isolation and Characterization}

Exosomes were purified from the conditioned medium (CM) of HepG2 cells using ultracentrifugation methods as described previously. ${ }^{31}$ Briefly, the CM was collected from HepG2 cells, and then centrifuged at $300 \times \mathrm{g}$ for $10 \mathrm{~min}, 2000 \times \mathrm{g}$ for 15 min, and $10,000 \times \mathrm{g}$ for $30 \mathrm{~min}$ to remove cell debris and additional cellular fragments. After that, the supernatants were ultracentrifuged at $120,000 \times \mathrm{g}$ for 70 minutes to isolated exosomes. The pelleted exosomes were then resuspended in $100 \mu \mathrm{L}$ PBS and stored at $-80^{\circ} \mathrm{C}$. Nanoparticle tracking analysis (NTA) was used to analyze the quantity and size of exosomes. A JSM-7800F transmission electron microscopy 
Table I Primer Sequences

\begin{tabular}{|l|l|l|}
\hline Name & & \multicolumn{1}{c|}{ Primer Sequences } \\
\hline Actin & $\begin{array}{l}\text { Forward } \\
\text { Reverse }\end{array}$ & $\begin{array}{l}\text { 5'-GTCCACCGCAAATGCTTCTA-3' } \\
\text { 5'-TGCTGTCACCTTCACCGTTC-3' }\end{array}$ \\
\hline hsa_circ_0074854 & $\begin{array}{l}\text { Forward } \\
\text { Reverse }\end{array}$ & $\begin{array}{l}\text { 5'-AAGGGAACCTTTCACTGGTCTG-3' } \\
\text { 5'-AGAGGCAGCATCTGGCTGAT-3' }\end{array}$ \\
\hline IL-10 & Forward & 5'-TGGAGGACTTTAAGGGTTACCTG-3' \\
& Reverse & 5'-TGTTTTCACAGGGAAGAAATCG-3' \\
\hline TNF-a & Forward & 5'-CTGTAGCCCATGTTGTAGCAAAC-3' \\
& Reverse &
\end{tabular}

(TEM, StarJoy, Japan) was utilized to identify the morphology of exosomes. The HepG2 cell-derived exosomes were analyzed by Western blotting using the following primary antibodies: anti-TSG101 and anti-CD63.

\section{Exosome Labeling and Uptake}

Exosomes secreted by the HepG2 cells were fluorescently labeled with PKH26 red membrane dye (Sigma Aldrich). After that, the labeled exosomes were co-cultured with macrophages for $24 \mathrm{~h}$ at $37^{\circ} \mathrm{C}$. DAPI (blue) was used to label nuclei. Later on, internalization of exosomes by macrophages was observed by a fluorescence microscope (Carl Zeiss, Jena, Germany).

\section{Macrophage Polarization Assay}

The macrophages were treated with $20 \mathrm{ng} / \mathrm{mL}$ IL-4 and 20 ng/mL IL-13 (for M2 polarization), or HepG2 cell-derived exosomes for $24 \mathrm{~h}$. The macrophages surface makers CD86 (M1 marker) and CD206 (M2 marker) were analyzed by flow cytometry (BD Biosciences).

\section{Transwell Assays}

Cell migration and invasion assays were analyzed using 24-well Transwell cell culture chambers (Corning) with or without precoated Matrigel. To explore the effect of hsa_circ_0074854 knockdown on cell migration and invasion, $2 \times 10^{4}$ transfected HCC cells were added into the upper chamber, and the bottom chamber was added with $600 \mu \mathrm{L}$ medium containing $10 \% \mathrm{FBS}$. In addition, to investigate the effect of M2 macrophages induced by HCC cellsecreted exosomes on migration and invasion of $\mathrm{HCC}$ cells, $2 \times 10^{4} \mathrm{HCC}$ cells were added into the upper chamber. Meanwhile, $1 \times 10^{4}$ macrophages were cocultured with exosomes, suspended in $600 \mu \mathrm{L}$ medium with $10 \%$ FBS and then added into the bottom chamber. After incubation at $37^{\circ} \mathrm{C}$ and $5 \% \mathrm{CO}_{2}$ for $24 \mathrm{~h}$, the cells on the lower surface of the chamber were stained with crystal violet for $30 \mathrm{~min}$. Subsequently, the migrated and invasive cells were captured using a fluorescence microscope (Leica, Buffalo Grove, IL, USA) in five random microscopic fields, and then the cells were counted.

\section{Animal Studies}

$\mathrm{BALB} / \mathrm{c}$ nude mice (4-5 weeks old) were purchased from the Vital River Laboratories (Beijing, China) and maintained under pathogen-free conditions following the recommended procedures of National Institutes of Health guide for the care and use of laboratory animals. All animal experiments were performed in accordance with a protocol approved by the Ethics Committee of the Union Hospital, Tongji Medical College, Huazhong University of Science and Technology (No. HU20190401X). Animals were divided randomly into seven groups: (I) control, circRNA-shRNA1 and circRNA-shRNA1 + lenti-HuR groups; (II) HepG2, HepG2 + Macrophages (HepG2 + M), HepG2 + exosomes-treated macrophages (HepG2 $+\mathrm{M}^{\mathrm{Exo}}$ ) and HepG2 $+\mathrm{M}^{\text {Exo-circRNA shRNA1 }}$ groups. Group I: 5 x $10^{6}$ HepG2 or HepG2 cells stably expressing hsa_circ_0074854-shRNA1 cells in $100 \mu \mathrm{L}$ PBS were injected into the left flank of nude mice. Group II: HepG2 cells plus different hsa_circ_0074854 levels exosomes-treated macrophages were subcutaneously injected into left flank of nude mice. The tumor size was measured every week and the volume was calculated by the following formula $\mathrm{V}=\left(\right.$ length $\mathrm{x}$ width ${ }^{2}$ )/ 2. After 3 weeks, the mice were sacrificed, and the tumors were removed. Immunohistochemistry (IHC) was applied to assess CD206 expression in tumor tissues. 


\section{Statistical Analysis}

Data are presented as the mean \pm standard deviation (S.D.). All statistical analyses were performed using GraphPad Prism software (version 7.0, La Jolla, CA, USA). Differences between three or more groups were analyzed by one-way analysis of variance (ANOVA) and Tukey's tests. Differences between two groups were analyzed by twotailed Student's $t$-test. The differences were considered significant at $* \mathrm{P}<0.05$. All data were repeated in triplicate.

\section{Results}

\section{Identification of DEcircRNAs in the HCC Tissues and Normal Tissues}

The GSE94508 and GSE97332 datasets were selected for screening the DEcircRNAs between HCC tissues and normal tissues. The upregulated and downregulated circRNAs in HCC tissues compared with normal tissues are shown in Figure 1A and B. A total of 287 and 896 DEcircRNAs were identified in the GSE94508 and GSE97332 datasets, respectively (Figure 1C and D). Using a Venn diagram, 6 overlapping upregulated circRNAs were identified in the GSE94508 and GSE97332 datasets (Figure 1E). The 6 upregulated overlapping DEcircRNAs included hsa_circ_0000517, hsa_circ_0004720, hsa circ 0003141, hsa_circ_0006913, hsa_circ_00074854, hsa_circ_0088046 (Figure 1F). The highest expression level of hsa_circ_00074854 was observed in HCC tissues.

\section{Hsa circ 00074854 Knockdown Suppressed Proliferation and Induced Apoptosis of HCC Cells}

To investigate the role of hsa circ 00074854 in HCC cells, we analyzed hsa_circ_00074854 levels in one human hepatic cell line L02, and four HCC cell lines HepG2, Huh-7, Hep3B2.1-7 and HCCLM3, by using RTqPCR. We found that hsa_circ_00074854 level was notably upregulated in HepG2, Huh-7 and HCCLM3 cells, compared with L02 cells (Figure 2A and Supplementary Figure S1A). Additionally, the level of hsa_circ_00074854 was significantly decreased in HepG2 and Huh-7 cells following transfection with hsa_circ_00074854-shRNA1 (Figure 2B). Moreover, hsa_circ_00074854 knockdown inhibited the proliferation and induced the apoptosis of HepG2 and Huh-7 cells (Figure 2C-F). Meanwhile, downregulation of hsa_circ_00074854 significantly decreased the expression of Bcl-2 and increased the expressions of active caspase 3 and PCDC5 in HepG2 and Huh-7 cells
(Figure $2 \mathrm{G}$ and $\mathrm{H}$ ). These results indicated that downregulation of hsa_circ_00074854 could inhibit proliferation and induced apoptosis of HCC cells.

\section{Hsa_circ_00074854 Knockdown Inhibited Migration, Invasion and EMT of HCC Cells}

Next, the role of hsa_circ_00074854 in the regulation of migration and invasion in $\mathrm{HCC}$ cells was determined using transwell assays. As indicated in Figure $3 \mathrm{~A}$ and $\mathrm{B}$ and Supplementary Figure S1B, downregulation of hsa circ 00074854 markedly inhibited the migration and invasion abilities of HepG2, Huh-7 and HCCLM3 cells, compared with the shRNA-NC group. In addition, downregulation of hsa circ_00074854 significantly upregulated the expression of E-cadherin and downregulated the expressions of vimentin and $\alpha$-SMA in HepG2 and Huh-7 cells (Figure 3C and D). These data suggested that downregulation of hsa circ_00074854 could inhibit migration, invasion and EMT of HCC cells.

\section{Hsa_circ_00074854 Physically Interacts with HuR in HCC Cells}

It has been shown that circRNAs can interact with RNAbinding proteins in cancers. ${ }^{32} \mathrm{HuR}$, as an RNA-binding protein, has been found to promote migration and invasion of cancer cells. ${ }^{33,34}$ Thus, we investigated whether hsacirc_00074854 could regulate HuR in HCC. Then, the interaction between hsa_circ_00074854 and HuR was assessed by RNA pulldown assay. As shown in Figure 4A, the HuR protein was pulled down by hsa_circ_00074854. In addition, FISH results revealed the co-localization of hsa circ 00074854 and HuR in the cytoplasm of HepG2 cells (Figure 4B). These data indicated a close relationship between hsa_circ_00074854 and HuR.

\section{Hsa_circ_00074854 Knockdown Inhibited the Migration, Invasion, and EMT of HepG2 Cells via HuR}

We next to investigate the effect of hsa_circ_00074854 on HuR expression. RT-qPCR results showed that hsacirc_00074854 levels had no change in HuR mRNA levels in HepG2 cells, but Western blot results presented a decreased protein expression of HuR when hsa_circ_00074854 was silenced (Figure 5A and B). In addition, the level of hsa_circ_00074854 was markedly increased in HepG2 cells transfected with lenti-hsa_circ_00074854 (Figure 5C). We then 

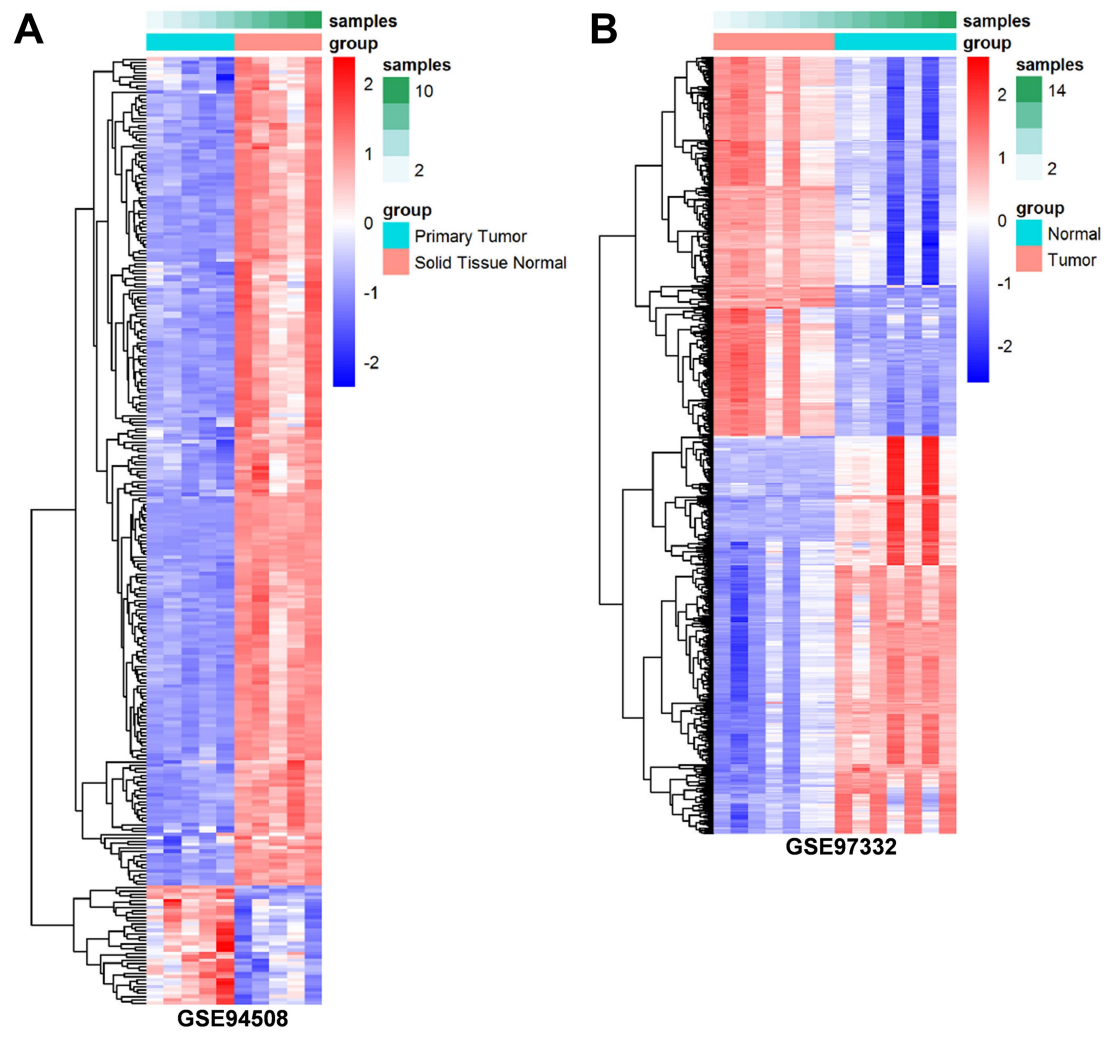
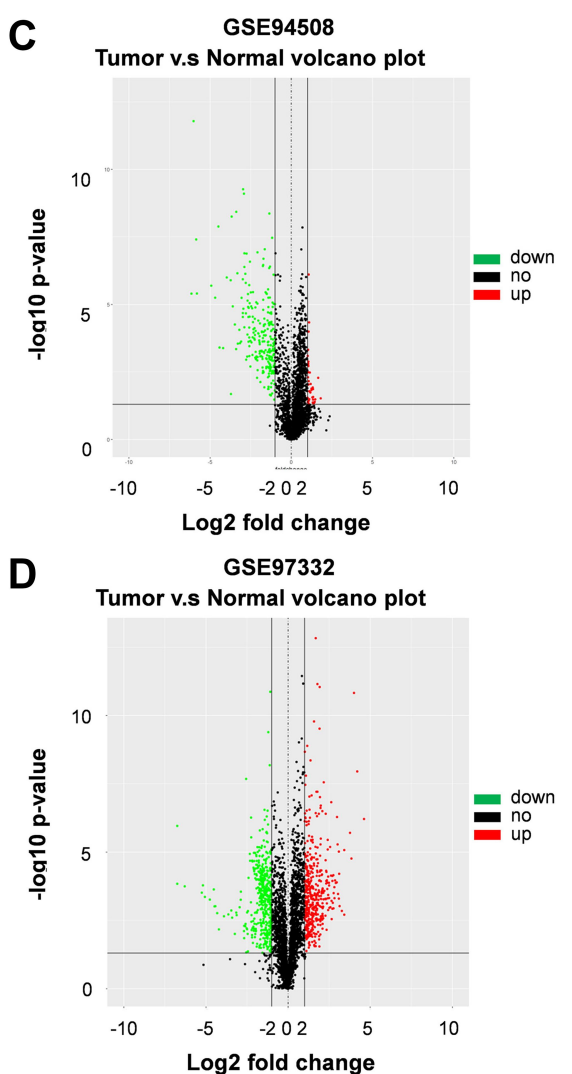

E

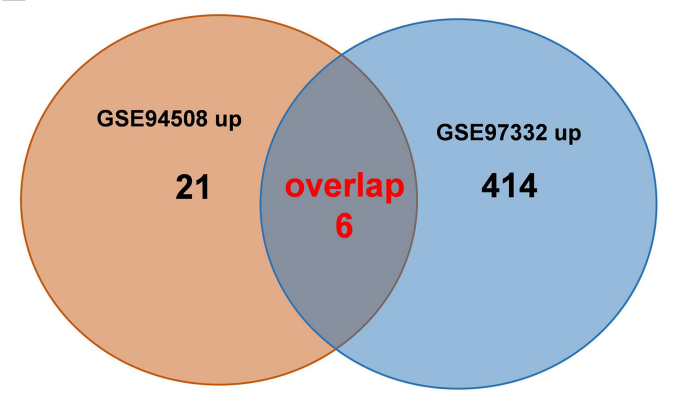

$\mathbf{F}$

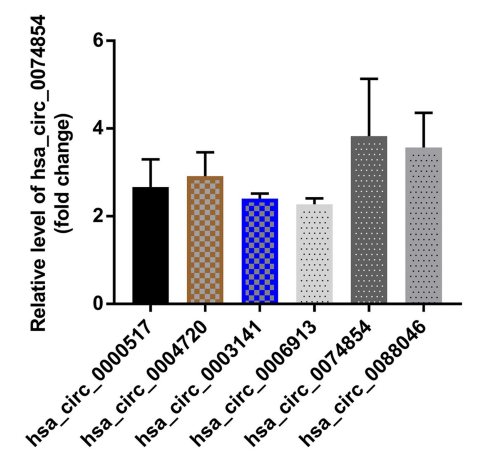

Figure I Identification of DEcircRNAs in the HCC tissues and normal tissues. (A and B) Heatmap showing the circRNA expression profiles of HCC tissues and normal tissues, downloaded from GSE94508 and GSE97332 datasets. (C and D) The volcano plot of DEcircRNAs from the GSE94508 and GSE97332 datasets. Red plots represented the upregulated circRNAs, green plots represented the downregulated circRNAs. (E) Venn diagram of overlapping upregulated DEcircRNAs from intersection of GSE94508 and GSE97332 databases. (F) 6 upregulated circRNAs from HCC tissues and normal tissues were identified using R language.

Abbreviations: HCC, hepatocellular carcinoma; DEcircRNAs, differentially expressed circRNAs.

incubated HepG2 cells with the protein synthesis inhibitor cycloheximide (CHX) and assessed the stability of HuR. As shown in Figure 5D, the stability of HuR protein increased when hsa_circ_00074854 was overexpressed and decreased when hsa_circ_00074854 was silenced. To illustrate whether HuR can mediate the migration, invasion and EMT of HepG2 cells, we established HuR knockdown and overexpressed HepG2 cells (Figure 5E). In addition, the results of transwell assays indicated that knockdown of HuR notably suppressed migration and invasion of HepG2 cells (Figure 5F and G). Moreover, downregulation of HuR significantly downregulated the levels of ZEB-1 and vimentin and upregulated the level of E-cadherin in HepG2 cells (Figure 5H). Next, we further investigate whether hsa_circ_00074854 knockdown inhibits the EMT of HepG2 cells via HuR. As indicated in Figure 5I, downregulation of hsa_circ_00074854 notably downregulated the levels of ZEB-1 and vimentin and upregulated the level of E-cadherin in HepG2 cells; however, these levels were markedly reversed by HuR overexpression. Furthermore, downregulation of hsa_circ_00074854 significantly induced the apoptosis of HepG2 cells; however, that effect was reversed by HuR overexpression (Figure 5J). These 


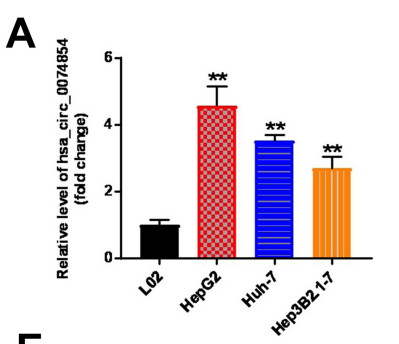

E
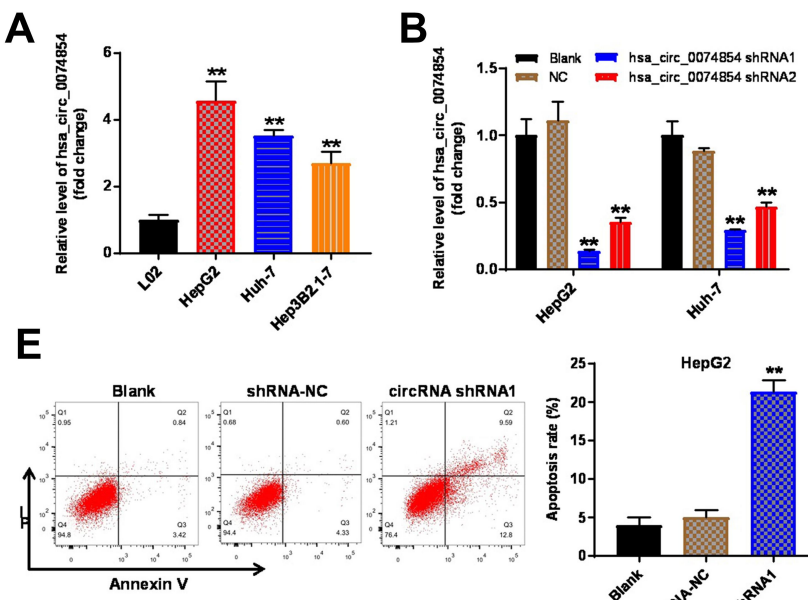
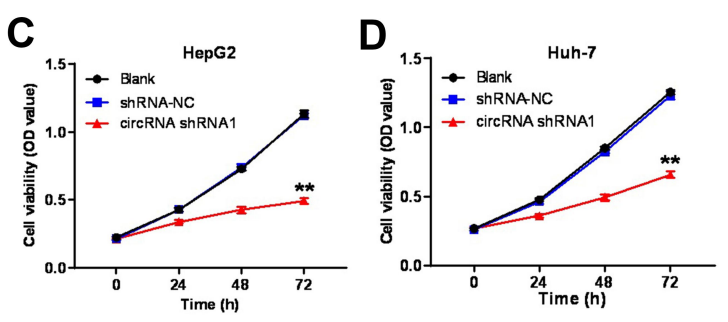

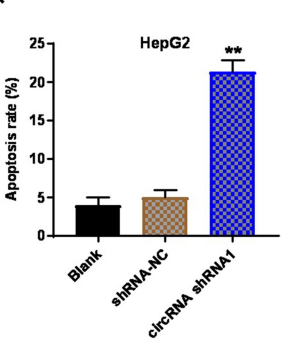

$\mathbf{F}$
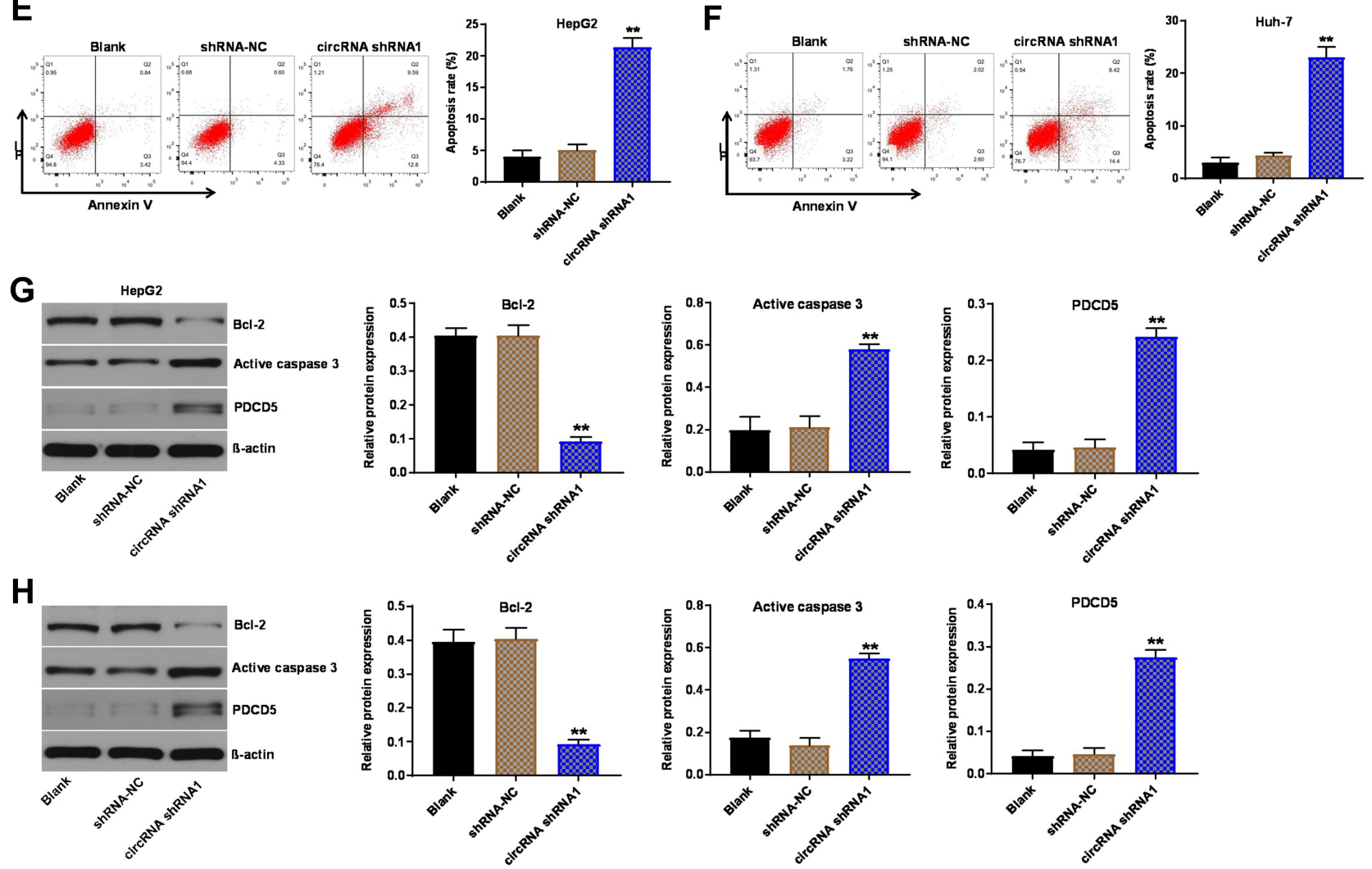

Figure 2 Downregulation of hsa_circ_00074854 inhibited proliferation and induced apoptosis of HCC cells. (A) The levels of hsa_circ_00074854 in HCC cell lines (HepG2, Huh-7, Hep3B2.I-7), normal human hepatic cell line L02 were detected by RT-qPCR. **P < 0.01 vs L02 group. (B) RT-qPCR analysis of hsa_circ_00074854 expression in HepG2 and Huh-7 cells transfected with hsa_circ_00074854 shRNAI or hsa_circ_00074854 shRNA2. **P < 0.0 I vs NC group. (C and D) The viability of HepG2 and Huh-7 cells transfected with hsa_circ_00074854 shRNAI detected by CCK-8 assay. *** $<0.01$ vs shRNA-NC group. (E and F) Apoptosis detected by Annexin $\mathrm{V}$ and PI double staining in HepG2 and Huh-7 cells transfected with hsa_circ_00074854 shRNAI. **P < 0.0 I vs shRNA-NC group. (G and $\mathbf{H})$ Western blot analysis of Bcl-2, active caspase 3 and PDCD5 in HepG2 and Huh-7 cells transfected with hsa_circ_00074854 shRNAI. **P < 0.01 vs shRNA-NC group.

Abbreviations: CCK-8, Cell Counting Kit-8; circRNA shRNAI, HepG2 or Huh-7 cells transfected with hsa_circ_00074854-shRNAI; HCC, hepatocellular carcinoma; PI, propidium iodide; RT-qPCR, reverse transcription quantitative real-time PCR; shRNA-NC, HepG2 or Huh-7 cells transfected with shRNA negative control.

results suggested that downregulation of hsa_circ_00074854 could inhibit the migration, invasion, and EMT of HepG2 cells via interacting with HuR.

\section{Hsa_circ_00074854 Knockdown Inhibited the Growth of HCC Cells in vivo via HuR}

We next investigated whether hsa_circ_00074854 knockdown could affect tumorigenicity in vivo. As revealed in Figure 6A$\mathrm{C}$ and Supplementary Figure S2A-C, downregulation of hsa circ_00074854 markedly decreased the tumor volume and tumor weight of HepG2 subcutaneous xenografts, compared with control group; however, these effects were markedly reversed by HuR overexpression. These data indicated that hsa_circ_00074854 knockdown could inhibit the growth of HCC cells in vivo.

\section{Exosomes with Downregulated hsa_circ_00074854 Suppressed Macrophage M2 Polarization in vitro}

Cancer-derived exosomes play an important role in establishing the tumor microenvironment. ${ }^{18}$ In this study, we 

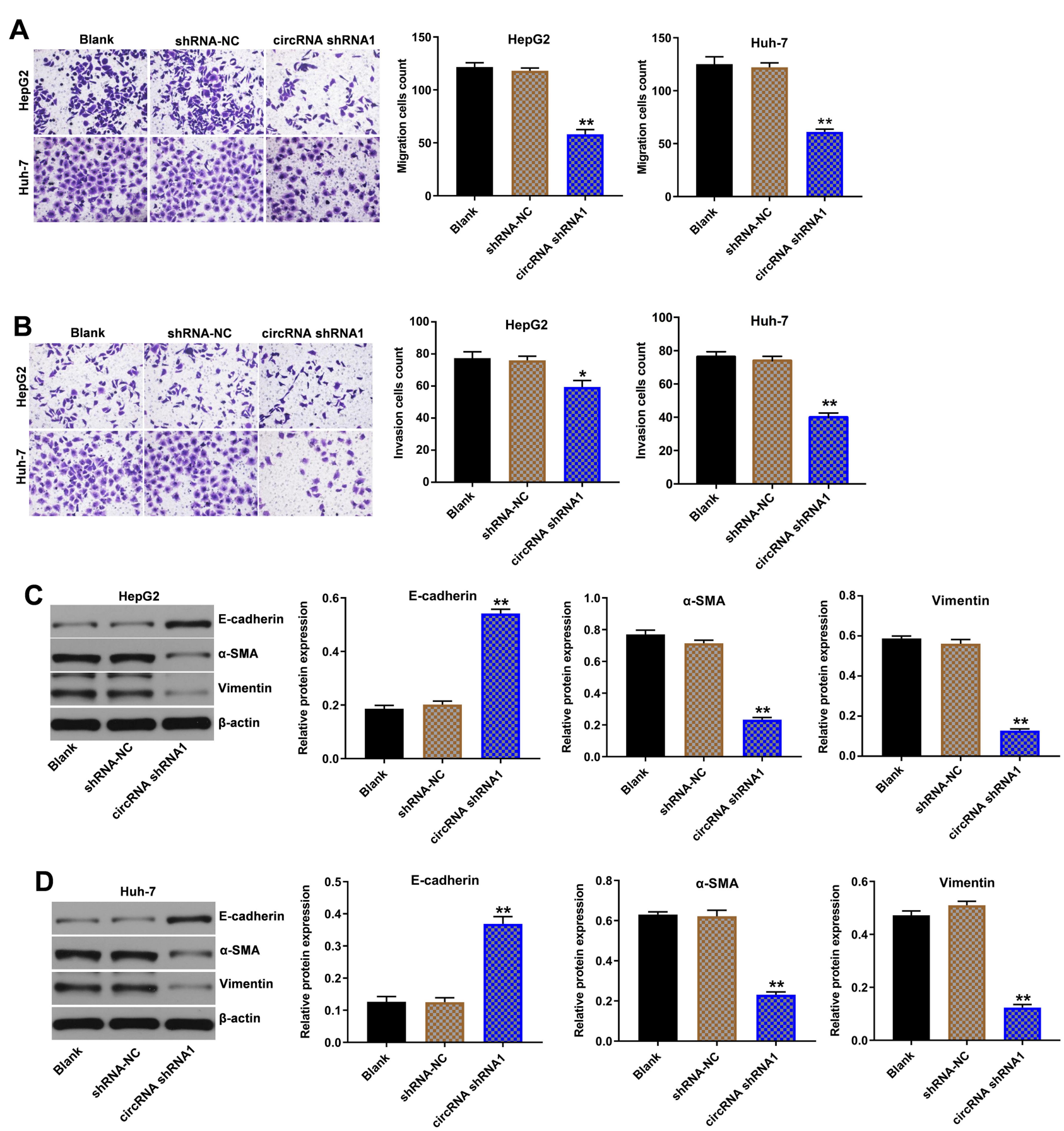

Figure 3 Downregulation of hsa circ 00074854 inhibited migration, invasion and EMT of HCC cells. (A) The migratory ability of HepG2 and Huh-7 cells transfected with hsa_circ_00074854 shRNAI detected by transwell migration assay. (B) The invasive ability of HepG2 and Huh-7 cells transfected with hsa_circ_00074854 shRNAI detected by transwell invasion assay. (C and $\mathbf{D})$ Western blot analysis of E-cadherin, $\alpha$-SMA and vimentin in HepG2 and Huh-7 cells transfected with hsa_circ_00074854 shRNAI. *P $<0.05$, **P $<0.01$ vs shRNA-NC group.

Abbreviations: CircRNA shRNAI, HepG2 or Huh-7 cells transfected with hsa_circ_00074854-shRNAI; HCC, hepatocellular carcinoma; shRNA-NC, HepG2 or Huh-7 cells transfected with shRNA negative control.

isolated exosomes from the conditioned medium (CM) of L02 and HepG2 cells. TEM and NTA were used to identify the determine the quantity and number of exosomes. As shown in Figure 7A and B, exosomes secreted from L02 and HepG2 cells were approximately $100 \mathrm{~nm}$ in diameter with typical cup-shaped morphology. In addition, exosomal markers, TSG101 and CD63, were identified using Western blot assay (Figure 7C). These data confirmed that the vesicles isolated from the $\mathrm{CM}$ were exosomes. Meanwhile, exosomal hsa_circ_00074854 was found to 

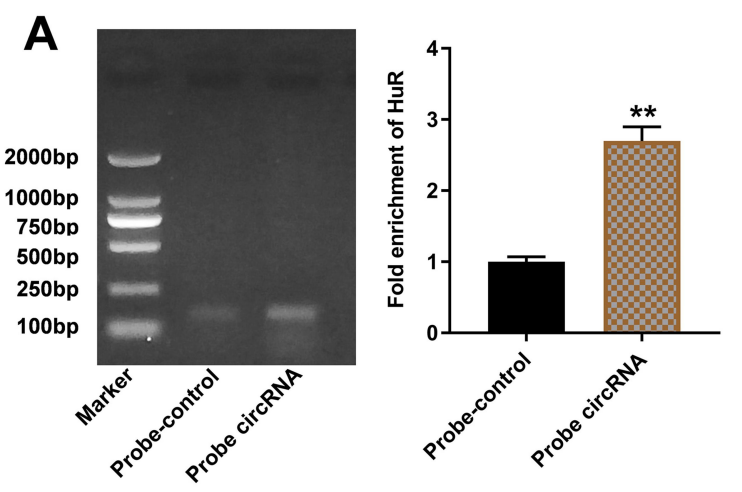

B

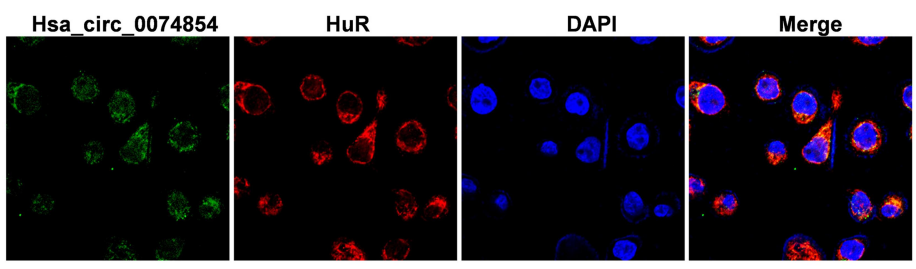

Figure 4 Hsa_circ_00074854 physically interacts with HuR in HCC cells. (A) Level of HuR pulled down by biotin-labeled hsa_circ_00074854. **P < $0.0 \mathrm{I}$ vs probe-control group. (B) Colocalization of hsa_circ_00074854 and HuR in HepG2 cells detected by FISH.

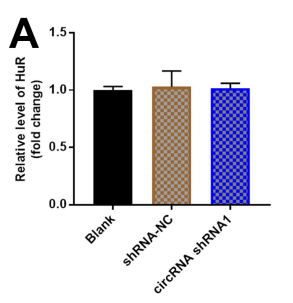

E

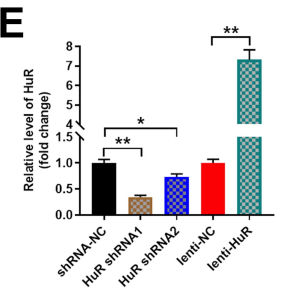

B

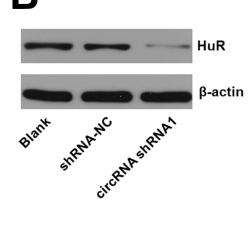

F

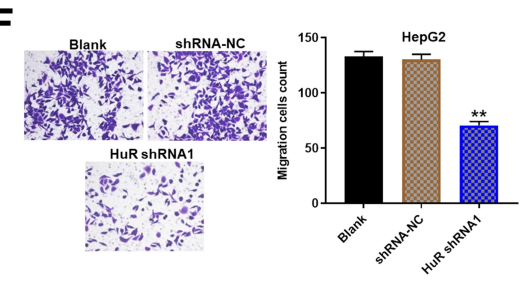

C

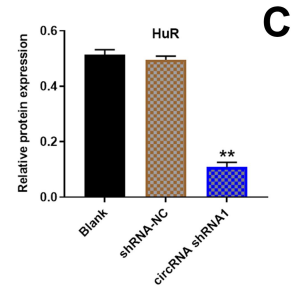

G2

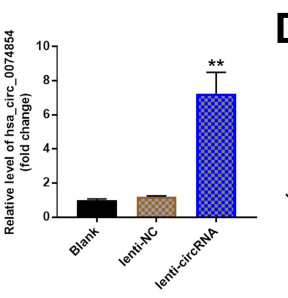

D

G

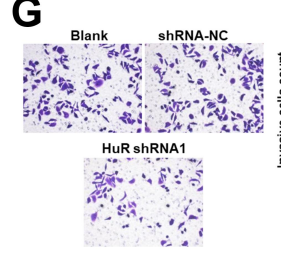

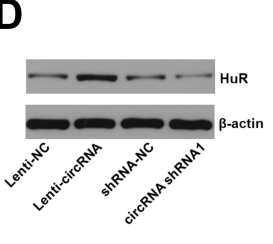
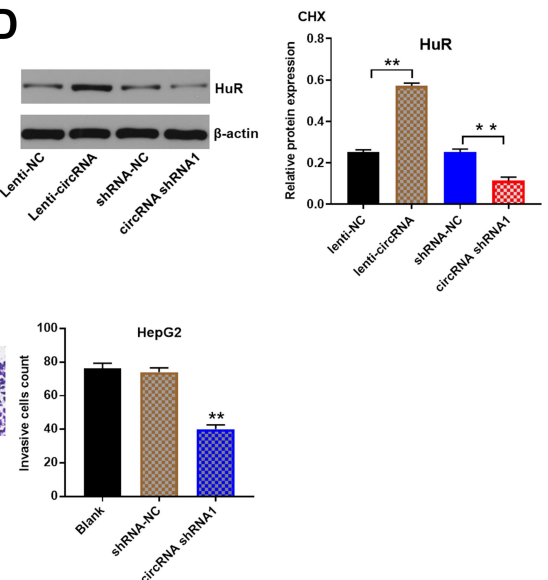

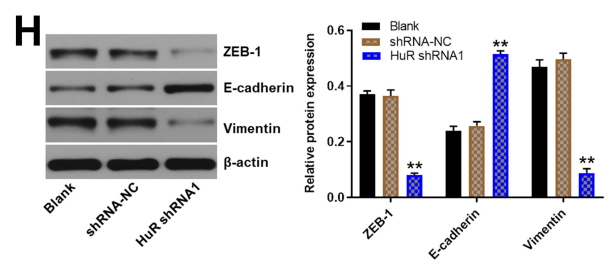

I
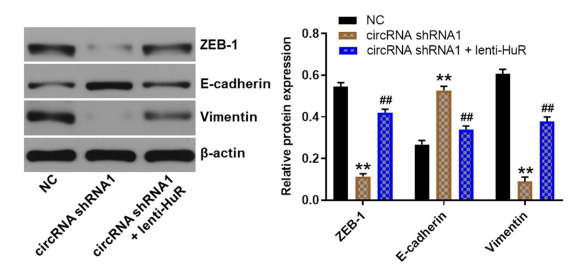

J
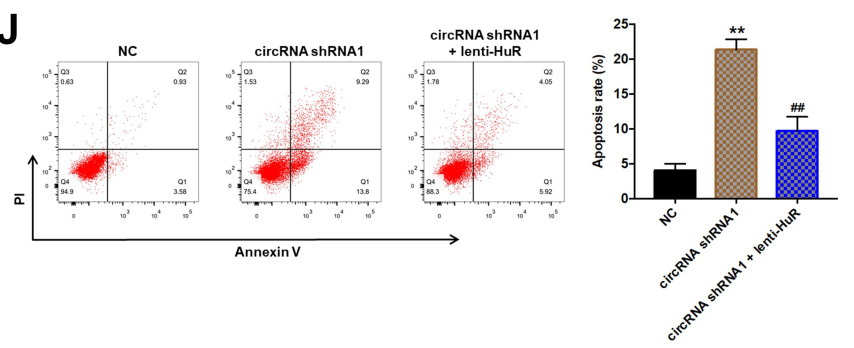

Figure 5 Downregulation of hsa_circ_00074854 inhibited the migration, invasion, and EMT of HepG2 cells via HuR. (A) Relative HuR mRNA levels were detected by RTqPCR in HepG2 cells with hsa circ 00074854 knockdown. (B) Western blot analysis for HuR protein levels in HepG2 cells transfected with hsa circ 00074854 shRNAI. **P $<0.01$ vs shRNA-NC group. (C) RT-qPCR analysis of hsa_circ_00074854 expression in HepG2 cells transfected with lenti-hsa_circ_00074854. $* * \bar{P}<0.01$ vs lenti-NC group. (D) HepG2 cells with hsa_circ_00074854 overexpression or knockdown were treated with $50 \mu g / \mathrm{mL} \mathrm{CHX}$ for $12 \mathrm{~h}$. Western blot assay was used to detect the expression of HuR in HepG2 cells. **P < 0.0I. (E) RT-qPCR analysis of HuR expression in HepG2 cells transfected with HuR shRNAI, HuR shRNA2 or lenti-HuR. *P < 0.05; $* * \mathrm{P}<0.01$. (F) The migratory ability of HepG2 cells transfected with HuR shRNAI detected by transwell migration assay. **P $<0.0 \mathrm{I}$ vs shRNA-NC group. (G) The invasive ability of HepG2 cells transfected with HuR shRNAI detected by transwell invasion assay. **P < 0.01 vs shRNA-NC group. (H) Western blot analysis of ZEB-I, E-cadherin and vimentin in HepG2 cells transfected with HuR shRNAI. **P $<0.01$ vs shRNA-NC group. (I) Western blot analysis of ZEB-I, E-cadherin and vimentin in HepG2 cells transfected with hsa_circ_00074854 shRNAI or hsa_circ_00074854 shRNAI plus lenti-HuR. **P < 0.01 vs NC group; ${ }^{\text {N }}$ < 0.0 l vs circRNA-shRNAI group. (J) Flow cytometry assay was used to determine cell apoptosis. $* * \mathrm{p}<0.0 \mathrm{I}$ vs NC group; ${ }^{\# \#} \mathrm{P}<0.01$ vs circRNA-shRNAI group.

Abbreviations: $\mathrm{CHX}$, cycloheximide; circRNA shRNAI, HepG2 cells transfected with hsa_circ_00074854-shRNAI; lenti-circRNA, HepG2 cells transfected with lenti-hsa _circ_00074854; shRNA-NC, HepG2 cells transfected with shRNA negative control. 

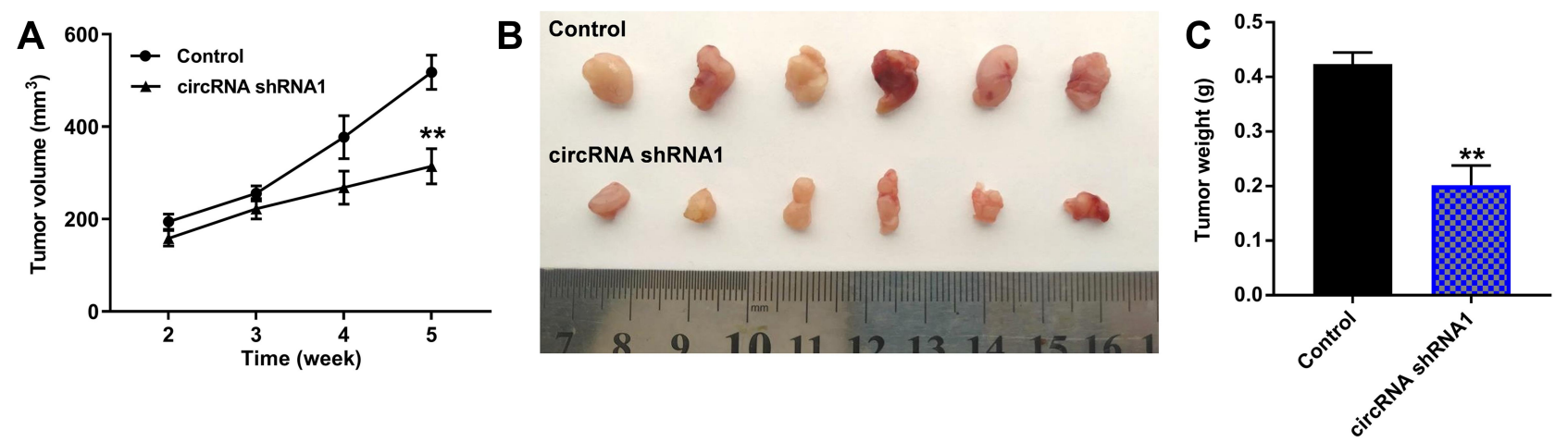

Figure 6 Downregulation of hsa_circ_00074854 inhibited the growth of HCC cells in vivo. Mice were subcutaneously implanted with hsa_circ_00074854 shRNAItransfected HepG2 cells. (A) Tumor volume was measured in different groups every week. (B) Representative image of xenograft tumors at day 35 . (C) The tumor weight was measured. ${ }^{*} \mathrm{P}<0.01$ vs control group.

Abbreviations: circRNA shRNAI, HepG2 cells transfected with hsa_circ_00074854-shRNAI.

be enriched in the CM of HepG2 cells, rather than L02 cells, as shown by the RT-qPCR analysis (Figure 7D).

It has been shown that circRNAs can be transferred from tumor cells to recipient cells via exosomes. ${ }^{35}$ Exosomes present in the tissue microenvironment, and TAMs are the most abundant tumor-infiltrating immune cells in tumor microenvironment. ${ }^{36}$ Therefore, we hypothesized that cancer-derived exosomal hsa_circ_00074854 participated in the communication between tumor cells and TAMs. To determine whether exosomes could be internalized by recipient cells, PKH26-label exosomes were co-cultured with macrophages (PMAdifferentiated human THP-1 monocytes). As revealed in Figure 7E, the uptake of exosomes was observed in macrophages after co-culture for $24 \mathrm{~h}$. In contrast, no PKH26 fluorescence dye was detected in macrophages without the treatment of exosomes (Figure 7E). Evidence has shown that cancer-derived exosomes can induce M2 macrophage polarization. ${ }^{37}$ Thus, macrophages were incubated with 25 or $50 \mu \mathrm{g} / \mathrm{mL}$ HepG2 cell-derived exosomes (IL-4/IL-13 treatment as a positive control), and the surface markers CD86 (M1 phenotype) and CD206 (M2 phenotype) were analyzed by flow cytometry. As shown in Figure 7F, macrophages exhibited a $\mathrm{CD} 86^{\text {low }} / \mathrm{CD} 206^{\text {high }}$ phenotype, when cells were incubated HepG2 cell-derived exosomes, suggesting that HepG2 cell-derived exosomes could induce the M2 polarization of macrophages. Next, we isolated the exosomes from the CM of HepG2 cells transfected with hsa_circ_00074854-shRNA1 and found that hsa_circ_00074854 levels were significantly decreased in exosomes with downregulated hsa_circ_00074854 (Figure $7 \mathrm{G})$. In addition, macrophages exhibited a CD86 ${ }^{\text {high} /}$
CD206 ${ }^{\text {low }}$ phenotype, when cells were incubated with exosomes containing lower hsa_circ_00074854 levels (Figure 7H). Moreover, in comparison with untreated macrophages, macrophages treated with HepG2 cellderived exosomes expressed markedly more M2 representative cytokine IL-10, but there were no significant changes of M1 representative cytokine TNF-a (Figure 7I). In contrast, macrophages incubated with HepG2 cellderived exosomes containing lower hsa_circ_00074854 levels showed notably reduced level of IL-10 compared to those incubated with HepG2 cell-derived exosomes (Figure 7I). Overall, exosomes with downregulated hsa_circ_00074854 could suppress macrophage M2 polarization in vitro.

\section{Exosomes with Downregulated} hsa_circ_00074854 Inhibited Macrophage M2 Polarization to Suppress Migration, Invasion and EMT in HepG2 Cells in vitro

To explore whether hsa_circ_00074854 knockdown could suppress migration, invasion and EMT of HepG2 cells by suppressing M2 macrophages polarization, macrophages treated with exosomes containing lower hsa_circ_00074854 levels were indirectly co-cultured with HepG2 cells (Figure 8A). The results of transwell migration and invasion assays showed that M2 macrophages induced by HepG2 cell-derived exosomes obviously promoted the migration and invasion of HepG2 cells; however, these effects were markedly reversed by exosomes containing lower hsa_circ_00074854 levels (Figure 8B). In addition, the levels of ZEB-1 and vimentin were increased, and the level of E-cadherin was decreased in 

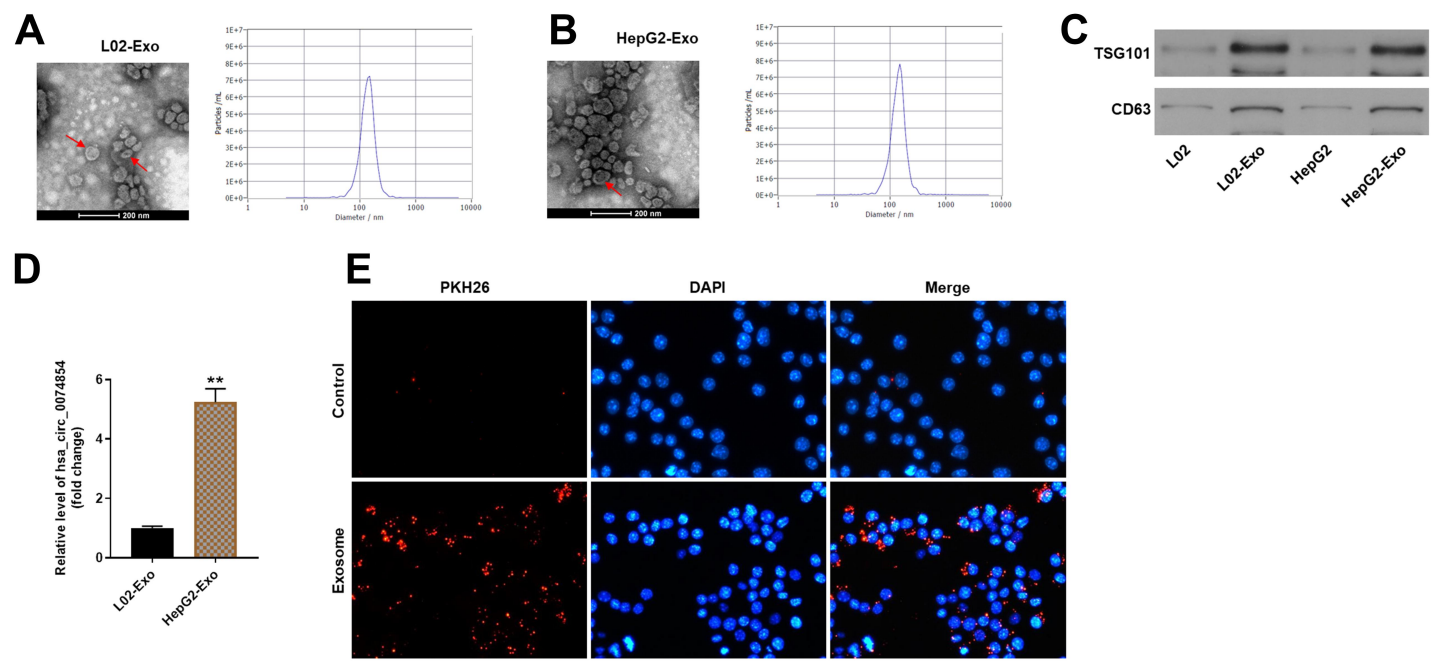

\section{$\mathbf{F}$}
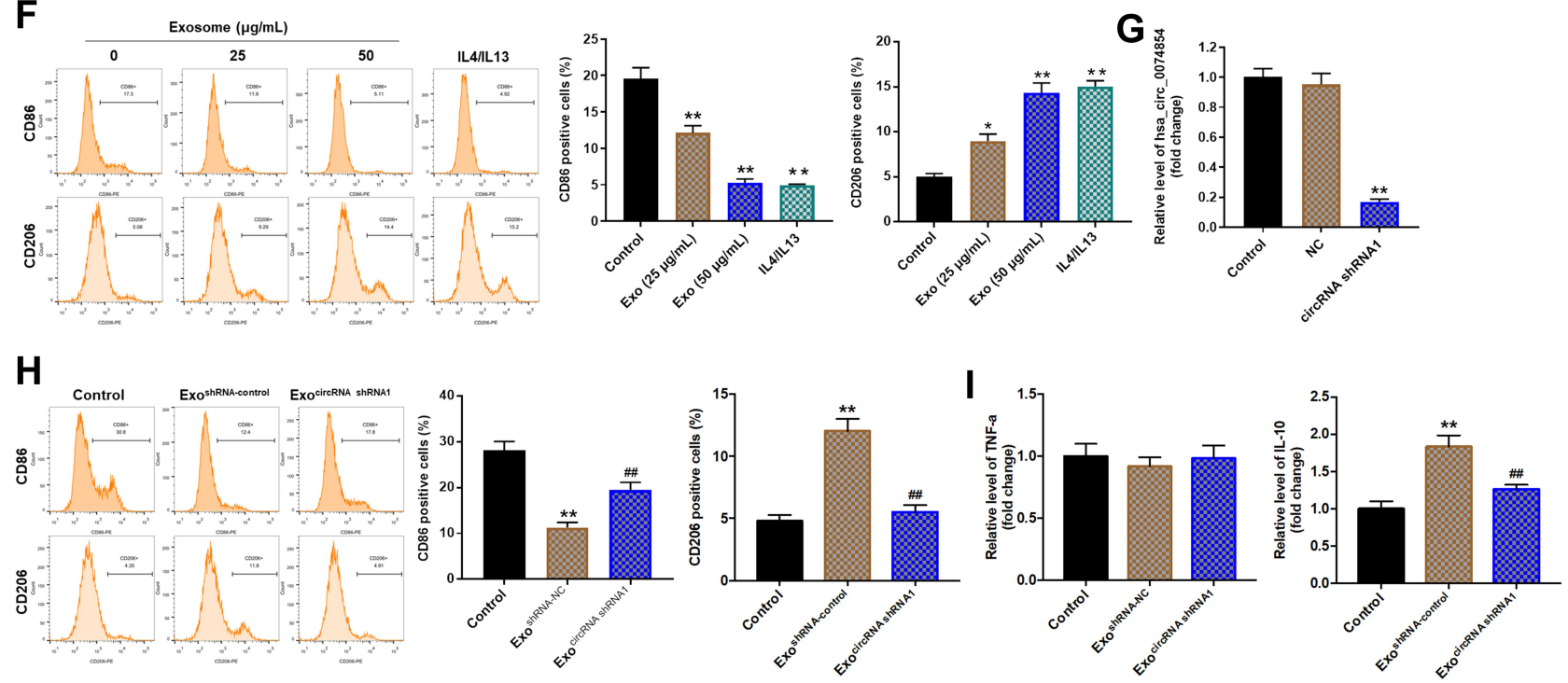

Figure 7 Exosomes with downregulated hsa_circ_00074854 suppressed macrophage M2 polarization in vitro. (A and B) TEM of L02 and HepG2 cell CM secreted exosomes (Red arrow, exosome). Scale bar, $200 \mathrm{~nm}$. NTA was used to detect the size and number of exosomes. (C) Western blot analysis for exosomal proteins TSGI0I and CD63. (D) The level of hsa_circ_00074854 was analyzed by RT-qPCR in exosomes isolated from L02 cells and HepG2 cells. **P < 0.01 vs L02-Exo group. (E) Confocal microscopy of the internalization of PKH26-labeled HepG2 cell-derived exosomes (red) by macrophages. (F) Macrophages were treated with exosomes $(0,25,50 \mu g / m L)$ isolated from CM of HepG2 cells. The percentage of CD86- and CD206-positive macrophages were analyzed by flow cytometry. *P < 0.05 , **P < 0.01 vs control group. (G) RT-qPCR analysis of hsa circ 00074854 levels in exosomes isolated from the CM of hsa circ 00074854 knockdown HepG 2 cells. **P < 0.01 compared with NC group. $(\mathbf{H})$ Flow cytometry analysis of the expressions of CD86/CD206 in macrophages treated with exosomes with downregulated hsa_circ_00074854 levels. **P < 0.01 vs Control group; ${ }^{\# \#} \mathrm{P}<0.01$ vs Exo ${ }^{\text {shRNA-NC }}$ group. (I) RT-qPCR analysis of the levels of IL-I0 and TNF-a in macrophages treated with exosomes with downregulated hsa_circ_00074854 levels. **P $<0.0$ I vs Control group; ${ }^{\# \#} \mathrm{P}<0.0$ I vs Exo ${ }^{\text {shRNA-NC }}$ group.

Abbreviations: CM, conditioned medium; Exo, exosomes; Exo ${ }^{\text {circRNA shRNAl }}$, exosomes derived from hsa_circ_00074854 shRNAI-transfected HepG2 cells; Exo ${ }^{\text {shRNA-NC, }}$ exosomes derived from shRNA NC-transfected HepG2 cells; NC, negative control.

HepG2 cells after co-culturing with M2 macrophages induced by HepG2 cell-derived exosomes (Figure 8C). In contrast, macrophages treated with exosomes containing lower hsa_circ_00074854 levels significantly downregulated the levels of ZEB-1 and vimentin and upregulated the level of E-cadherin in HepG2 cells (Figure 8C). Collectively, exosomes with downregulated hsa_circ_00074854 could inhibit macrophage M2 polarization to suppress migration, invasion and EMT in HCC cells.
Exosomes with Downregulated hsa_circ_00074854 Suppressed the Growth of HepG2 Cells via Inhibiting M2 Macrophage Polarization in vivo

To investigate the function of exosomes with downregulated hsa_circ_00074854 in inhibiting macrophage M2 polarization, which suppress the progression of HCC in vivo, HepG2 cells and macrophages treated with exosomes containing lower hsa_circ_00074854 levels were 


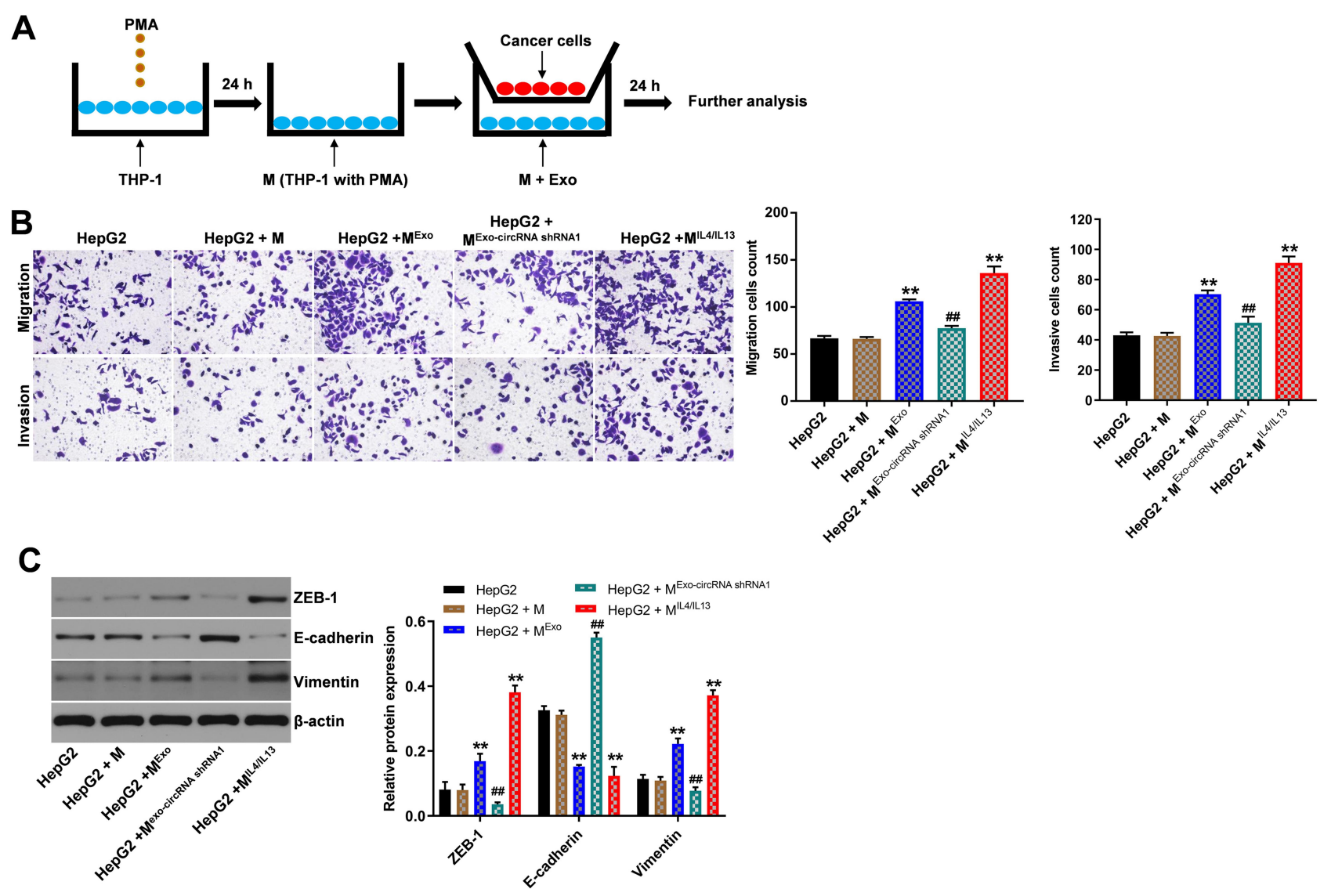

Figure 8 Exosomes with downregulated hsa_circ_00074854 inhibited macrophage M2 polarization to suppress migration, invasion and EMT in HepG2 cells in vitro. (A) Schema for representing the experiment procedures. (B) The migration and invasion abilities of HepG2 cells co-cultured with macrophages treated with exosomes were assessed by the transwell co-culture system. (C) HepG2 cells incubated with the supernatants of macrophages treated with exosomes. Western blot analysis of ZEB-I, E-cadherin and vimentin in HepG2 cells. **P $<0.01$ vs HepG2 + M group; ${ }^{\#} \mathrm{P}<0.01$ compared with HepG2 $+\mathrm{M}^{\mathrm{Exo}}$ group.

Abbreviations: $M$, macrophages (PMA-differentiated human THP-I monocytes); $M^{\text {Exo }}$, macrophages co-cultured with exosomes derived from HepG2 cells; $M^{\text {Exo-circRNA }}$ shRNAI, macrophages co-cultured with exosomes derived from hsa_circ_00074854 shRNAI-transfected HepG2 cells; $M^{\text {IL4/LII3 }}$, macrophages co-cultured with IL4 and ILI3 $(20 \mathrm{ng} / \mathrm{mL})$; PMA, phorbol- 12 myristate- 13 acetate.

subcutaneously injected into nude mice. As shown in Figure $9 \mathrm{~A}-\mathrm{C}$, the tumor volume and tumor weight in the

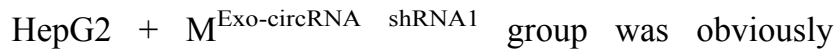
decreased, compared with the HepG $2+M^{\text {Exo }}$ group. In addition, macrophages treated with HepG2 cell-derived exosomes upregulated the levels of CD206 and IL-10 in the xenografts; however, these effects were reversed by exosomes containing lower hsa_circ_00074854 levels (Figure 9D-F). Moreover, the level of TNF-a in HepG2 $+\mathrm{M}^{\text {Exo-circRNA shRNA1 }}$ group was not different compared with that in HepG2 $+M^{\text {Exo }}$ group. Meanwhile, macrophages treated with exosomes containing lower hsa_circ_00074854 levels downregulated the levels of ZEB-1 and vimentin and upregulated the expression of E-cadherin in the xenografts (Figure 9G). These data indicated that hsa_circ_00074854 knockdown inhibited HCC tumorigenesis via suppressing macrophage M2 polarization.

\section{Discussion}

CircRNAs have been found to involve in various biological processes, including cell metastasis and invasion. ${ }^{9}$ In this study, we found that hsa_circ_00074854 is upregulated in $\mathrm{HCC}$ tissues. Downregulation of hsa_circ_00074854 inhibited the migration and invasion of HCC cells in vitro and in vivo. Mechanistically, hsa_circ_00074854 knockdown reduced the stability of HuR protein, and then suppressed EMT in HCC cells. Additionally, HCC cell-secreted hsa_circ_00074854 can be delivered to macrophages via exosomes. Meanwhile, exosomes with downregulated hsa_circ_00074854 could inhibit macrophage M2 polarization, thereby suppressing migration, invasion and EMT in HCC cells. These data indicated that hsa_circ_00074854 knockdown could inhibit HCC cell migration and invasion via functioning within cells and affecting the tumor microenvironment. 
A

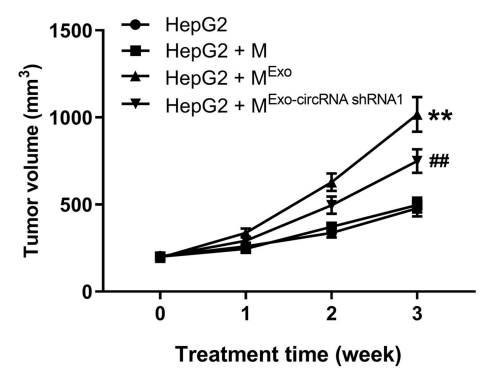

D

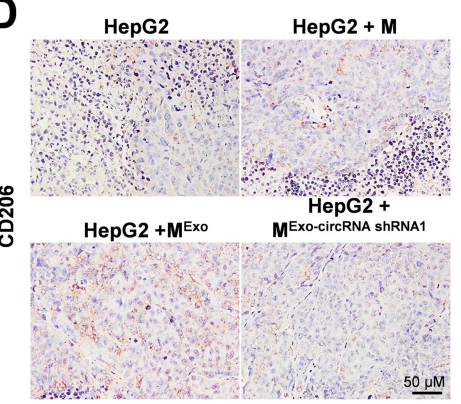

F

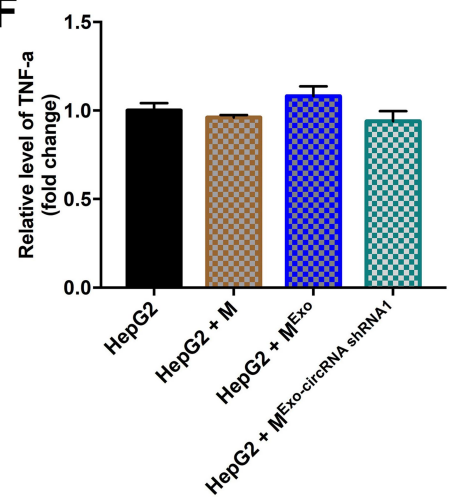

B HepG2
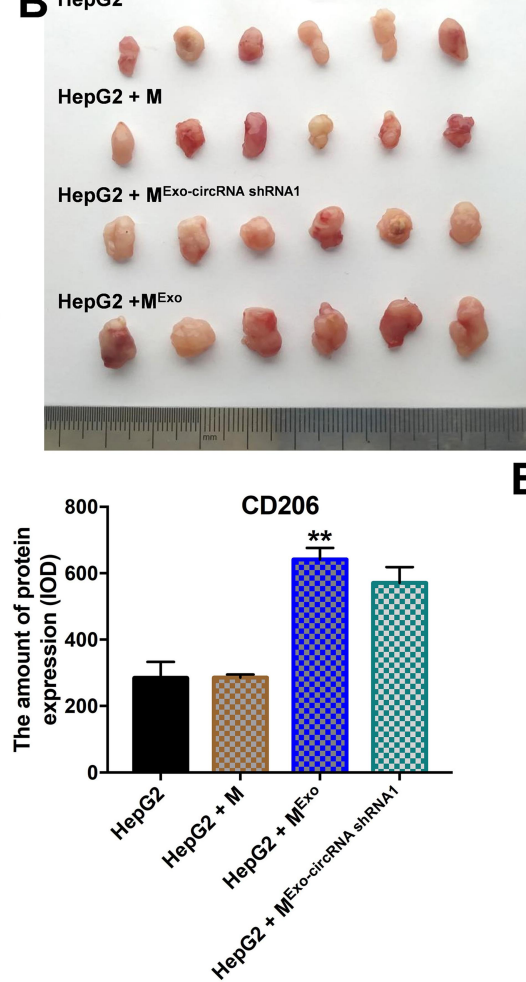

G

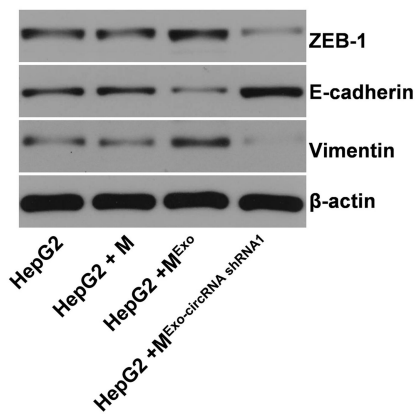

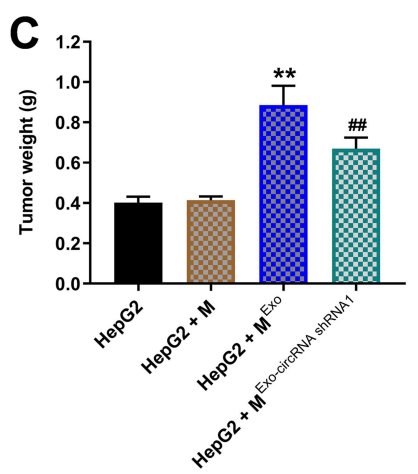

$\mathbf{E}$

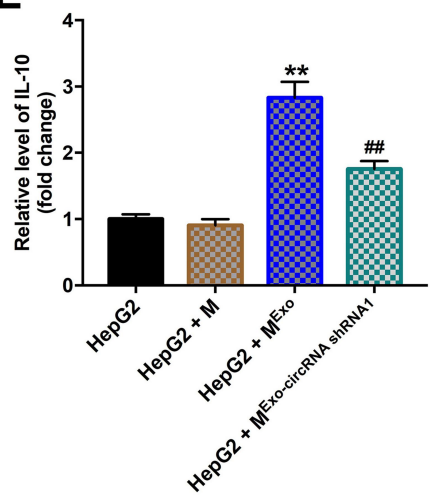

H

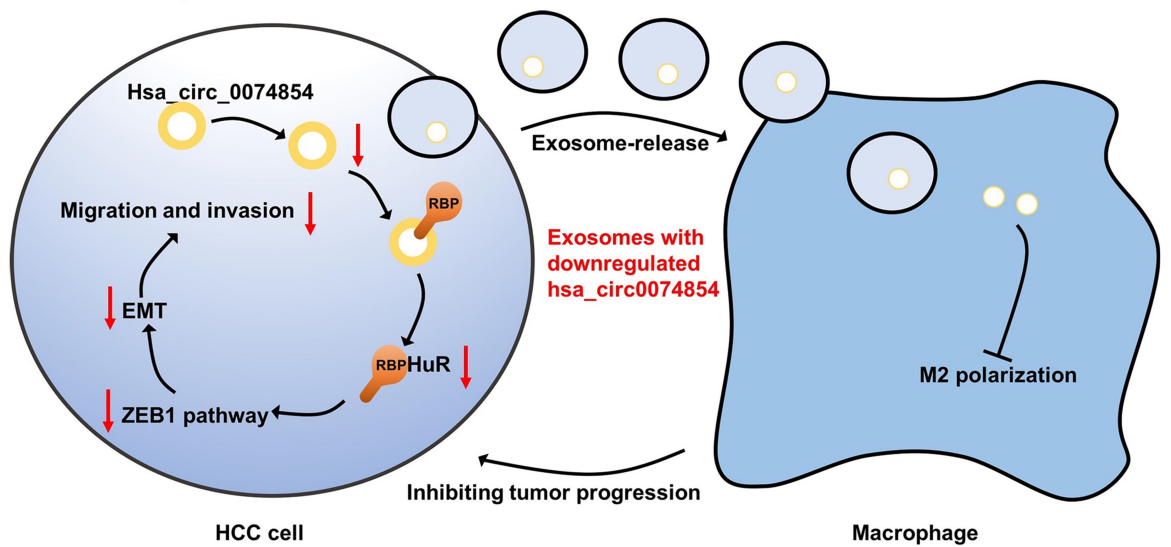

Figure 9 Exosomes with downregulated hsa_circ_00074854 suppressed the growth of HepG2 cells via inhibiting M2 macrophage polarization in vivo. Animals were subcutaneously injected with HepG2 cells and different hsa_circ_00074854 levels exosomes-treated macrophages. (A) Tumor volume was measured in different groups every week. (B) Representative image of xenograft tumors at day 2I. (C) The tumor weight was measured. (D) Immunohistochemistry of CD206. (E and F) RT-qPCR assay was used to detect the level of IL-I0 and TNF-a in tumor tissues. (G) Western blot analysis of ZEB-I, E-cadherin and vimentin in tumor tissues. (H) Schematic model of hsa_circ_00074854 functions in tumor progression. $* * \mathrm{P}<0.01$ vs HepG2 + M group; ${ }^{\# \# P}<0.01$ compared with HepG2 $+M^{\mathrm{Exo}}$ group.

Abbreviations: $M$, macrophages (PMA-differentiated human THP-I monocytes); $M^{\text {Exo }}$, macrophages co-cultured with exosomes derived from HepG2 cells; $M^{\text {Exo-circRNA }}$ shRNAI, macrophages co-cultured with exosomes derived from hsa_circ_00074854 shRNAI-transfected HepG2 cells. 
HuR, an RNA binding protein (RBP), is known to stabilize mRNAs by binding to adenylate/uridylate-rich elements (AREs) and preventing gene degradation. ${ }^{38,39}$ Evidences have been shown that HuR is highly abundant in many cancers, including HCC. ${ }^{40,41}$ In addition, elevated HuR expression is associated with poor prognosis of cancer patients. ${ }^{42}$ Many circRNAs are predicted to interact with RBPs. ${ }^{43}$ Chen et al found that circAGO2 can bind to HuR to facilitate its activation and then promote cancer progression. ${ }^{44}$ In this study, RNA pull-down analysis revealed that hsa_circ_00074854 interacted with HuR. Interestingly, when investigating the regulation of hsa_circ_00074854 on HuR expression, we found that hsa_circ_00074854 knockdown decreased the HuR protein level but had no effect on its mRNA level. Meanwhile, we discovered that hsa_circ_00074854 knockdown regulated HuR level in HCC cells via reducing HuR protein stability. Previous study reported that HuR could stabilize ZEB1 mRNA level, and then induce EMT in cigarette smoke extract-treated bronchial epithelial cells. ${ }^{45}$ Consistently, our results showed that downregulation of HuR decreased the expression of ZEB1 in HCC cells and then inhibited EMT in HCC cells. Meanwhile, hsa_circ_00074854 knockdown could block the ZEB1 signaling, thus suppressing EMT. Collectively, our data indicated that hsa_circ_00074854 knockdown inhibited migration, invasion and EMT in HCC cells via reducing HuR protein stability and inhibition of ZEB1 signaling (Figure 9G).

Our data have found that hsa_circ_00074854 could regulate the proliferation, migration and invasion of $\mathrm{HCC}$ cells. In addition, it has been shown that circRNAs can be transported by exosomes from cancer cells to recipient cells within TME. ${ }^{13,46}$ This process is crucial for tumor progression as well. ${ }^{47}$ The communication between HCC cells and TME is an important mechanism regulating tumor progression. ${ }^{47}$ Thus, another important finding from our study was that exosomes with downregulated hsa_circ_00074854 can be delivered to macrophages to inhibit macrophage M2 polarization. Previous study showed that tumor exosomes carry circRNAs and are released into TME. $^{48}$ TME is a complex system that is composed of a variety of cells and cytokines. ${ }^{49}$ Importantly, exosomes can create an immune suppression environment for tumor cells to survive via communication between tumor cells and surrounding recipient cells. ${ }^{50}$ TAMs are the most abundant immune cells in TME, which promote tumor cell migration and invasion mostly by secreting inflammatory cytokines. ${ }^{24,51,52}$ Meanwhile, M2 macrophage-like TAMs are known to promote tumor proliferation and metastasis. ${ }^{53,54}$ Evidence has shown that IL-10 secreted by M2 macrophage could promote the migration and invasion of tumor cells. ${ }^{55,56}$ Consistently, in this study, we found that HCC cell-derived exosomes induced M2 macrophage activation, which promoted migration, invasion and EMT in HCC cells. In contrast, exosomes with downregulated hsa_circ_00074854 suppressed macrophage M2 polarization, which inhibited migration, invasion and EMT in HCC cells both in vitro and in vivo. These data suggested that exosomes with downregulated hsa_circ_00074854 could inhibit macrophage M2 polarization to suppress migration and invasion in HCC cells (Figure 9G). Collectively, exosomes serve as an important messenger that mediated the cross-talk between tumor cells and TAMs.

\section{Conclusion}

In summary, we provided the evidence that hsa_circ_0074854 knockdown inhibited the migration and invasion of HCC cells by binding to HuR, thus inhibiting its stability and by suppressing exosomes-mediated macrophage M2 polarization. These findings may help to understand the diagnosis and treatment of HCC.

\section{Data Sharing Statement}

The data that support the findings of this study are available from the corresponding author upon reasonable request.

\section{Acknowledgment}

These authors contributed equally to this work and should be considered as co-first authors: Yong Wang, Rongfen Gao, and Jinpeng Li.

\section{Disclosure}

The authors declare that they have no conflicts of interest for this work.

\section{References}

1. Ozer Etik D, Suna N, Boyacioglu AS. Management of hepatocellular carcinoma: prevention, surveillance, diagnosis, and staging. Exp Clin Transplant. 2017;15(Suppl 2):31-35. doi:10.6002/ect.TOND16.L9

2. Siegel RL, Miller KD, Jemal A. Cancer statistics, 2016. CA Cancer J Clin. 2016;66(1):7-30. doi:10.3322/caac.21332

3. Yang JD, Hainaut P, Gores GJ, et al. A global view of hepatocellular carcinoma: trends, risk, prevention and management. Nat Rev Gastroenterol Hepatol. 2019;16(10):589-604. doi:10.1038/s41575019-0186-y 
4. Rasool M, Rashid S, Arooj M, et al. New possibilities in hepatocellular carcinoma treatment. Anticancer Res. 2014;34(4):1563-1571.

5. Higashi T, Friedman SL, Hoshida Y. Hepatic stellate cells as key target in liver fibrosis. Adv Drug Deliv Rev. 2017;121:27-42.

6. Heimbach JK, Kulik LM, Finn RS, et al. AASLD guidelines for the treatment of hepatocellular carcinoma. Hepatology. 2018;67 (1):358-380. doi:10.1002/hep.29086

7. Ren Z, Ma X, Duan Z, Chen X. Diagnosis, therapy, and prognosis for hepatocellular carcinoma. Anal Cell Pathol. 2020;2020:8157406. doi:10.1155/2020/8157406

8. Tsuchiya N, Sawada Y, Endo I, et al. Biomarkers for the early diagnosis of hepatocellular carcinoma. World $J$ Gastroenterol. 2015;21(37):10573-10583. doi:10.3748/wjg.v21.i37.10573

9. Wang L, Long H, Zheng Q, et al. Circular RNA circRHOT1 promotes hepatocellular carcinoma progression by initiation of NR2F6 expression. Mol Cancer. 2019;18(1):119. doi:10.1186/s12943-0191046-7

10. Memczak S, Jens M, Elefsinioti A, et al. Circular RNAs are a large class of animal RNAs with regulatory potency. Nature. 2013;495 (7441):333-338. doi:10.1038/nature11928

11. Zhang X, Xu Y, Qian Z, et al. circRNA_104075 stimulates YAP-dependent tumorigenesis through the regulation of HNF4a and may serve as a diagnostic marker in hepatocellular carcinoma. Cell Death Dis. 2018;9(11):1091. doi:10.1038/s41419-018-1132-6

12. Zhang S, Liu Y, Liu Z, et al. CircRNA_0000502 promotes hepatocellular carcinoma metastasis and inhibits apoptosis through targeting microRNA-124. J BUON. 2019;24(6):2402-2410.

13. Huang XY, Huang ZL, Huang J, et al. Exosomal circRNA-100338 promotes hepatocellular carcinoma metastasis via enhancing invasiveness and angiogenesis. J Exp Clin Cancer Res. 2020;39(1):20. doi:10.1186/s13046-020-1529-9

14. Kalluri R. The biology and function of exosomes in cancer. J Clin Invest. 2016;126(4):1208-1215. doi:10.1172/JCI81135

15. Dilsiz N. Role of exosomes and exosomal microRNAs in cancer. Future Sci OA. 2020;6(4):Fso465. doi:10.2144/fsoa-2019-0116

16. Li S, Li Y, Chen B, et al. exoRBase: a database of circRNA, lncRNA and mRNA in human blood exosomes. Nucleic Acids Res. 2018;46 (D1):D106-d112. doi:10.1093/nar/gkx891

17. Atienzar-Aroca S, Flores-Bellver M, Serrano-Heras G, et al. Oxidative stress in retinal pigment epithelium cells increases exosome secretion and promotes angiogenesis in endothelial cells. $J$ Cell Mol Med. 2016;20(8):1457-1466. doi:10.1111/jcmm.12834

18. Kahlert C, Kalluri R. Exosomes in tumor microenvironment influence cancer progression and metastasis. J Mol Med. 2013;91(4):431-437. doi:10.1007/s00109-013-1020-6

19. Wang H, Lu Z, Zhao X. Tumorigenesis, diagnosis, and therapeutic potential of exosomes in liver cancer. J Hematol Oncol. 2019;12 (1):133. doi:10.1186/s13045-019-0806-6

20. Ahmadi M, Rezaie J. Tumor cells derived-exosomes as angiogenenic agents: possible therapeutic implications. J Transl Med. 2020;18 (1):249. doi:10.1186/s12967-020-02426-5

21. Ghayad SE, Rammal G, Ghamloush F, et al. Exosomes derived from embryonal and alveolar rhabdomyosarcoma carry differential miRNA cargo and promote invasion of recipient fibroblasts. Sci Rep 2016;6:37088. doi:10.1038/srep37088

22. Li J, Li Z, Jiang P, et al. Circular RNA IARS (circ-IARS) secreted by pancreatic cancer cells and located within exosomes regulates endothelial monolayer permeability to promote tumor metastasis. J Exp Clin Cancer Res. 2018;37(1):177. doi:10.1186/s13046-018-0822-3

23. Ma YY, He XJ, Wang HJ, et al. Interaction of coagulation factors and tumor-associated macrophages mediates migration and invasion of gastric cancer. Cancer Sci. 2011;102(2):336-342. doi:10.1111/j.13497006.2010.01795.x

24. Lan J, Sun L, Xu F, et al. M2 macrophage-derived exosomes promote cell migration and invasion in colon cancer. Cancer Res. 2019;79 (1):146-158. doi:10.1158/0008-5472.CAN-18-0014
25. Wang X, Luo G, Zhang K, et al. Hypoxic tumor-derived exosomal miR-301a mediates M2 macrophage polarization via PTEN/PI3K $\gamma$ to promote pancreatic cancer metastasis. Cancer Res. 2018;78 (16):4586-4598. doi:10.1158/0008-5472.CAN-17-3841

26. Zheng P, Chen L, Yuan X, et al. Exosomal transfer of tumor-associated macrophage-derived miR-21 confers cisplatin resistance in gastric cancer cells. J Exp Clin Cancer Res. 2017;36(1):53. doi:10.1186/s13046-017-0528-y

27. Bardi GT, Smith MA, Hood JL. Melanoma exosomes promote mixed M1 and M2 macrophage polarization. Cytokine. 2018;105:63-72. doi:10.1016/j.cyto.2018.02.002

28. Schmall A, Al-Tamari HM, Herold S, et al. Macrophage and cancer cell cross-talk via CCR2 and CX3CR1 is a fundamental mechanism driving lung cancer. Am J Respir Crit Care Med. 2015;191 (4):437-447. doi:10.1164/rccm.201406-1137OC

29. Yang L, Dong Y, Li Y, et al. IL-10 derived from M2 macrophage

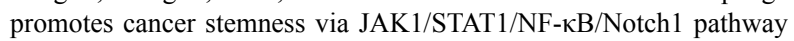
in non-small cell lung cancer. Int J Cancer. 2019;145(4):1099-1110. doi:10.1002/ijc.32151

30. Huang X, Li Z, Zhang Q, et al. Circular RNA AKT3 upregulates PIK3R1 to enhance cisplatin resistance in gastric cancer via miR-198 suppression. Mol Cancer. 2019;18(1):71. doi:10.1186/s12943-0190969-3

31. Greening DW, Xu R, Ji H, Tauro BJ, Simpson RJ. A protocol for exosome isolation and characterization: evaluation of ultracentrifugation, density-gradient separation, and immunoaffinity capture methods. Methods Mol Biol. 2015;1295:179-209.

32. Dong W, Dai ZH, Liu FC, et al. The RNA-binding protein RBM3 promotes cell proliferation in hepatocellular carcinoma by regulating circular RNA SCD-circRNA 2 production. EBioMedicine. 2019;45:155-167. doi:10.1016/j.ebiom.2019.06.030

33. Dormoy-Raclet V, Ménard I, Clair E, et al. The RNA-binding protein HuR promotes cell migration and cell invasion by stabilizing the beta-actin mRNA in a U-rich-element-dependent manner. Mol Cell Biol. 2007;27(15):5365-5380. doi:10.1128/MCB.00113-07

34. Hu YP, Jin YP, Wu XS, et al. LncRNA-HGBC stabilized by HuR promotes gallbladder cancer progression by regulating miR-502-3p/ SET/AKT axis. Mol Cancer. 2019;18(1):167. doi:10.1186/s12943019-1097-9

35. Shi X, Wang B, Feng X, et al. circRNAs and exosomes: a mysterious frontier for human cancer. Mol Ther Nucleic Acids. 2020;19:384-392. doi:10.1016/j.omtn.2019.11.023

36. Yin Z, Ma T, Huang B, et al. Macrophage-derived exosomal microRNA-501-3p promotes progression of pancreatic ductal adenocarcinoma through the TGFBR3-mediated TGF- $\beta$ signaling pathway. $J$ Exp Clin Cancer Res. 2019;38(1):310. doi:10.1186/s13046-0191313-x

37. Pritchard A, Tousif S, Wang Y, et al. Lung tumor cell-derived exosomes promote M2 macrophage polarization. Cells. 2020;9(5):1303. doi:10.3390/cells9051303

38. Chatterji P, Rustgi AK. RNA binding proteins in intestinal epithelial biology and colorectal cancer. Trends Mol Med. 2018;24(5):490-506. doi:10.1016/j.molmed.2018.03.008

39. Brennan CM, Gallouzi IE, Steitz JA. Protein ligands to HuR modulate its interaction with target mRNAs in vivo. J Cell Biol. 2000;151 (1):1-14. doi:10.1083/jcb.151.1.1

40. Kakuguchi W, Nomura T, Kitamura T, et al. Suramin, screened from an approved drug library, inhibits HuR functions and attenuates malignant phenotype of oral cancer cells. Cancer Med. 2018;7 (12):6269-6280. doi:10.1002/cam4.1877

41. Zhu H, Berkova Z, Mathur R, et al. HuR suppresses fas expression and correlates with patient outcome in liver cancer. Mol Cancer Res. 2015;13(5):809-818. doi:10.1158/1541-7786.MCR-14-0241

42. Song X, Shi X, Li W, Zhang F, Cai Z. The RNA-binding protein HuR in digestive system tumors. Biomed Res Int. 2020;2020:9656051. doi:10.1155/2020/9656051 
43. Hentze MW, Preiss PT. Circular RNAs: splicing's enigma variations. EMBO j. 2013;32(7):923-925. doi:10.1038/emboj.2013.53

44. Chen Y, Yang F, Fang E, et al. Circular RNA circAGO2 drives cancer progression through facilitating HuR-repressed functions of AGO2-miRNA complexes. Cell Death Differ. 2019;26 (7):1346-1364. doi:10.1038/s41418-018-0220-6

45. Sun J, Gu X, Wu N, et al. Human antigen $R$ enhances the epithelial-mesenchymal transition via regulation of ZEB-1 in the human airway epithelium. Respir Res. 2018;19(1):109. doi:10.1186/ s12931-018-0805-0

46. Bian X, Xiao YT, Wu T, et al. Microvesicles and chemokines in tumor microenvironment: mediators of intercellular communications in tumor progression. Mol Cancer. 2019;18(1):50. doi:10.1186/ s12943-019-0973-7

47. Pascut D, Pratama MY, Vo NVT, Masadah R, Tiribelli C. The crosstalk between tumor cells and the microenvironment in hepatocellular carcinoma: the role of exosomal microRNAs and their clinical implications. Cancers (Basel). 2020;12(4):823. doi:10.3390/cancers12040823

48. Shao Y, Lu B. The crosstalk between circular RNAs and the tumor microenvironment in cancer metastasis. Cancer Cell Int. 2020;20:448. doi:10.1186/s12935-020-01532-0

49. Wang J, Li D, Cang H, Guo B. Crosstalk between cancer and immune cells: role of tumor-associated macrophages in the tumor microenvironment. Cancer Med. 2019;8(10):4709-4721. doi:10.1002/ cam4.2327

50. Bae S, Brumbaugh J, Bonavida B. Exosomes derived from cancerous and non-cancerous cells regulate the anti-tumor response in the tumor microenvironment. Genes Cancer. 2018;9(3-4):87-100. doi:10.18632/genesandcancer.172
51. Chen Y, Song Y, Du W, et al. Tumor-associated macrophages: an accomplice in solid tumor progression. J Biomed Sci. 2019;26(1):78. doi:10.1186/s12929-019-0568-z

52. Wei C, Yang C, Wang S, et al. Crosstalk between cancer cells and tumor associated macrophages is required for mesenchymal circulating tumor cell-mediated colorectal cancer metastasis. Mol Cancer. 2019;18(1):64. doi:10.1186/s12943-019-0976-4

53. Yamaguchi T, Fushida S, Yamamoto Y, et al. Tumor-associated macrophages of the M2 phenotype contribute to progression in gastric cancer with peritoneal dissemination. Gastric Cancer. 2016;19 (4):1052-1065. doi:10.1007/s10120-015-0579-8

54. Yin $\mathrm{M}$, Li X, Tan S, et al. Tumor-associated macrophages drive spheroid formation during early transcoelomic metastasis of ovarian cancer. J Clin Invest. 2016;126(11):4157-4173. doi:10.1172/ JCI87252

55. Qi L, Yu H, Zhang Y, et al. IL-10 secreted by M2 macrophage promoted tumorigenesis through interaction with JAK2 in glioma. Oncotarget. 2016;7(44):71673-71685. doi:10.18632/ oncotarget. 12317

56. Liu Q, Yang C, Wang S, et al. Wnt5a-induced M2 polarization of tumor-associated macrophages via IL-10 promotes colorectal cancer progression. Cell Commun Signal. 2020;18(1):51. doi:10.1186/ s12964-020-00557-2
International Journal of Nanomedicine

\section{Publish your work in this journal}

The International Journal of Nanomedicine is an international, peerreviewed journal focusing on the application of nanotechnology in diagnostics, therapeutics, and drug delivery systems throughout the biomedical field. This journal is indexed on PubMed Central, MedLine, CAS, SciSearch ${ }^{\mathbb{R}}$, Current Contents ${ }^{\mathbb{R}} /$ Clinical Medicine, $^{2}$

\section{Dovepress}

Journal Citation Reports/Science Edition, EMBase, Scopus and the Elsevier Bibliographic databases. The manuscript management system is completely online and includes a very quick and fair peer-review system, which is all easy to use. Visit http://www.dovepress.com/ testimonials.php to read real quotes from published authors. 